\title{
Confidence Intervals for Assessing Non-Inferiority with Assay Sensitivity in a Three-Arm Trial with Normally Distributed Endpoints
}

\author{
Niansheng Tang * ${ }^{\mathbb{C}}$ and Fan Liang \\ Yunnan Key Laboratory of Statistical Modeling and Data Analysis, Yunnan University, Kunming 650091, China; \\ fional1217@sina.com \\ * Correspondence: nstang@ynu.edu.cn
}

Citation: Tang, N.; Liang, F. Confidence Intervals for Assessing

Non-Inferiority with Assay

Sensitivity in a Three-Arm Trial with Normally Distributed Endpoints. Mathematics 2022, 10, 167. https:// doi.org/10.3390/math10020167

Academic Editors: María del Carmen Pardo and Ying Lu

Received: 6 December 2021 Accepted: 28 December 2021 Published: 6 January 2022

Publisher's Note: MDPI stays neutral with regard to jurisdictional claims in published maps and institutional affiliations.

Copyright: (c) 2022 by the authors Licensee MDPI, Basel, Switzerland This article is an open access article distributed under the terms and conditions of the Creative Commons Attribution (CC BY) license (https:// creativecommons.org/licenses/by/ $4.0 /)$.

\begin{abstract}
Various approaches including hypothesis test and confidence interval (CI) construction have been proposed to assess non-inferiority and assay sensitivity via a known fraction or prespecified margin in three-arm trials with continuous or discrete endpoints. However, there is little work done on the construction of the non-inferiority margin from historical data and simultaneous generalized CIs (SGCIs) in a three-arm trial with the normally distributed endpoints. Based on the generalized fiducial method and the square-and-add method, we propose two simultaneous CIs for assessing non-inferiority and assay sensitivity in a three-arm trial. For comparison, we also consider the Wald-type Bonferroni simultaneous CI and parametric bootstrap simultaneous CI. An algorithm for evaluating the optimal sample size for attaining the pre-specified power is given. Simulation studies are conducted to investigate the performance of the proposed CIs in terms of their empirical coverage probabilities. An example taken from the mildly asthmatic study is illustrated using the proposed simultaneous CIs. Empirical results show that the proposed generalized fiducial method and the square-and-add method behave better than other two compared CIs.
\end{abstract}

Keywords: assay sensitivity; confidence interval; fiducial approach; square-and-add method; three-arm non-inferiority trial

\section{Introduction}

In modern clinical trials, it is extremely interesting to demonstrate whether a new treatment that is less toxic, less expensive or easier to administer is not inferior to an expensive and technical pathological reference treatment by more than a pre-specified margin. This is usually called a two-arm non-inferiority trial that does not include a placebo. Two-arm non-inferiority trials have been widely studied. For example, see [1-3]. However, two-arm non-inferiority trials have some shortcomings, such as the selection of the non-inferiority margin and the assessment of assay sensitivity (i.e., the ability to differentiate between an effective treatment and a less effective or ineffective treatment). If ethically acceptable and practically feasible, it is generally recognized that three-arm non-inferiority trials including a placebo can surmount the difficulties mentioned above [4].

There has been a growing interest in developing statistical inference on three-arm non-inferiority trials. For example, see [5], and [6] for the fractional margin, i.e., the noninferiority margin is taken as a pre-specified fraction of unknown effect size of reference treatment. In particular, Tang, Yu and Tang [7] developed the exact and approximate unconditional test approaches for the assessment of the non-inferiority in a three-arm trial with binary endpoints. However, the aforementioned literature did not consider the assessment of assay sensitivity. To this end, simultaneously assessing non-inferiority and assay sensitivity via a fixed margin has received considerable attention in recent years. For example, Hide and Tango [8] investigated simultaneous testing for both non-inferiority and assay sensitivity via a pre-specified margin, which is defined as a difference between two treatment effects in the considered historical placebo-controlled trial with normally 
distributed endpoints in the presence of homoscedasticity. Here endpoint represents the observation of random variable, and the normally distributed endpoint means that endpoint comes from a normal distribution. Hida and Tango's method may be improper due to the usage of the same margins for testing both non-inferiority and assay sensitivity. To solve the problem, Kwong et al. [9] considered a modified version of Hida and Tango's testing procedure based on different margins for assessing both non-inferiority and assay sensitivity. Mütze, Munk and Friede [10] presented a Wald-type test procedure for assessing both non-inferiority and assay sensitivity in a three-arm trial with negative binomially distributed endpoints.

The aforementioned literature mainly focuses on the non-inferiority testing problem in a three-arm trial. However, there is little work on simultaneously considering the construction of non-inferiority margin from the historical data and confidence interval for simultaneously testing both non-inferiority and assay sensitivity. Moreover, it is difficult to invert to find simultaneous confidence intervals for simultaneously testing both non-inferiority and assay sensitivity based on the aforementioned test procedures. To address these issues, here we consider the construction problem of simultaneous confidence intervals (CIs) for assessing both non-inferiority and assay sensitivity based on the square-and-add method and the generalized fiducial method in a three-arm trial.

CI construction via the square-and-add method [11], which is also called the method of variance estimates recovery, has received considerable attention over the past years in that it does not depend on the large sample theory and has a computationally simple and closed expression. For example, Li et al. [12] proposed an interval for the difference between two poisson rates based on the square-and-add method. Tang et al. [13] proposed five simultaneous CIs for risk differences in stratified matched-pair designs via the squareand-add method. Tang et al. [14] studied the problem of CI construction for the difference between two correlated proportions in the presence of incomplete paired binary data based on the square-and-add method. Zhang and Tang [15] extended the square-and-add method to CI construction of difference between two correlated areas under ROC curves in a matched-pair experiment. However, to our knowledge, there is little work done on simultaneous CI construction for assessing both non-inferiority and assay sensitivity in a three-arm trial using the square-and-add method.

Generalized CIs (GCIs) via generalized fiducial method [16] are widely studied in that it has been shown to be a useful tool for making inference in many practical problems. For example, see [17,18]. In particular, Schaarschmidt [19] discussed simultaneous CI construction for multiple comparisons among expected values of log-normal variables via the generalized fiducial method; Gamalo et al. [20] investigated the generalized CI construction problem for assessing non-inferiority in a three-arm trial with normally distributed endpoints in the presence of heteroscedasticity. However, Gamalo et al. [20] did not consider the assay sensitivity assessment problem. Moreover, to our knowledge, there is little work developed on simultaneous GCIs for assessing both non-inferiority and assay sensitivity in a three-arm trial with normally distributed endpoints. Hence, this paper proposes two simultaneous GCIs for simultaneously assessing non-inferiority and assay sensitivity by incorporating generalized fiducial pivotal quantity of [21] and the square-and-add approach in a three-arm non-inferiority trial with normally distributed endpoints, and presents their corresponding algorithms for evaluating GCIs. For comparison, we also consider Waldtype Bonferroni simultaneous CIs and parametric bootstrap simultaneous CIs introduced in [22]. In addition, we also discuss the problem of sample size determination, and present approximate formulae for determining sample size for attaining a pre-specified power.

The rest of this paper is organized as follows. Section 2 introduces the hypothesis testing problem for assessing both non-inferiority and assay sensitivity in a three-arm trial, discusses the problem of non-inferiority margin construction from historical data, presents four simultaneous CIs for assessing both non-inferiority and assay sensitivity based on generalized fiducial method, square-and-add approach, Wald-type Bonferroni method and parametric bootstrap method and gives the sample size formulae. Simulation studies and 
an example from the mildly asthmatic study are illustrated in Section 3. A brief discussion is given in Section 4. Technical details are presented in the Appendix A.

\section{Materials and Methods}

Following [9], we consider a one-way fixed effect model for endpoints in a three-arm non-inferiority trial with experimental, reference and placebo treatments. For notational simplicity, we denote experimental, reference and placebo treatments as E, R and P, respectively. Throughout this paper, we assume

$$
X_{i j}=\mu_{i}+\epsilon_{i j}, \quad i=\mathrm{E}, \mathrm{R}, \mathrm{P}, j=1, \ldots, n_{i},
$$

where $X_{i j}$ is the endpoint of the $j$ th individual for the $i$ th treatment, $\mu_{i}$ is the fixed effect of the $i$ th treatment, measurement errors $\epsilon_{i j} \stackrel{\text { i.i.d }}{\sim} \mathcal{N}\left(0, \sigma_{i}^{2}\right)$, and $n_{i}$ is the sample size of the $i$ th treatment for $i=\mathrm{E}, \mathrm{R}, \mathrm{P}$. Without loss of generality, it is assumed that a larger value of $\mu_{i}$ indicates the better efficacy for the $i$ th treatment. Under the above assumption, we have

$$
X_{i j} \stackrel{\text { i.i.d }}{\sim} \mathcal{N}\left(\mu_{i}, \sigma_{i}^{2}\right), \quad i=\mathrm{E}, \mathrm{R}, \mathrm{P}, j=1, \ldots, n_{i},
$$

To assess the non-inferiority (NI) and the assay sensitivity in a three-arm trial, Kwong et al. [9] considered the following modified HT hypotheses:

$$
\left\{\begin{array}{lll}
H_{\mathrm{NI}}^{0}: \mu_{E}-\mu_{R} \leq-r \Delta & \text { versus } & H_{\mathrm{NI}}^{1}: \mu_{E}-\mu_{R}>-r \Delta, \\
K_{\mathrm{AS}}^{0}: \mu_{R}-\mu_{P} \leq \Delta & \text { versus } & K_{\mathrm{AS}}^{1}: \mu_{R}-\mu_{P}>\Delta,
\end{array}\right.
$$

where $\Delta>0$ is a pre-specified margin, and $r$ is some pre-specified constant and satisfies the restricted condition: $0<r \leq 1$. In particular, when $r=1$, the hypotheses considered above are just those given in [8]. When $r<1$, the testing procedure for hypotheses (3) is more stringent than the testing procedure of [8] because of requiring assay sensitivity to be established. Clearly, if $H_{\mathrm{NI}}^{0}$ and $K_{\mathrm{AS}}^{0}$ are simultaneously rejected by some two-tailed test at the significance level $\alpha$ or two one-tailed tests at the significance level $\alpha / 2$, we may draw a conclusion: $\mu_{P}+\Delta<\mu_{R}<\mu_{E}+r \Delta$, which indicates that (i) the NI of experimental treatment to reference one with respect to $r \Delta$ and (ii) the superiority of reference treatment to placebo by more than $\Delta$. Here $\Delta$ is the entire effect size of reference treatment over placebo, and $\Delta_{\mathrm{NI}}=r \Delta$ is the NI margin showing the NI of experimental treatment to reference one.

Generally, one can construct two appropriate statistics for testing hypotheses $H_{\mathrm{NI}}^{0}$ and $K_{\mathrm{AS}}^{0}$. Here, rather than hypothesis testing, we consider the problem of constructing simultaneous CIs for $\phi=\mu_{E}-\mu_{R}$ and $\psi=\mu_{R}-\mu_{P}$ due to the duality of hypothesis testing and CI estimation. If the lower limits of the resultant $100(1-\alpha) \%$ one-sided simultaneous CIs for $\phi$ and $\psi$ are larger than $-r \Delta$ and $\Delta$, respectively, we can simultaneously reject the null hypotheses $H_{\mathrm{NI}}^{0}$ and $K_{\mathrm{AS}}^{0}$ at the significance level $\alpha$. However, in clinical studies, $\Delta_{\mathrm{NI}}$ is usually unknown. In what follows, we consider the construction problem of the NI margin, denoted as $\Delta_{\mathrm{NI}}$, via the lower bound of the credible interval of treatment effect of reference treatment in historical trials.

\subsection{Construction of NI Margin}

In what follows, a historical-trial-based approach and the generalized fiducial method are adopted to determine the NI margin $\Delta_{\mathrm{NI}}$. To this end, we first consider the generalized fiducial CI construction problem as follows.

Let $S \in \mathcal{R}^{k}$ be a random vector whose distribution is indexed by parameter vector $\xi \in \mathcal{R}^{p}$. Suppose we are interested in making inference on $\theta=\pi(\xi) \in \mathcal{R}^{q}$. Let $S^{*}$ be an independent copy of $S$, and $s$ and $s^{*}$ represent the observed values of $S$ and $S^{*}$, respectively. A generalized fiducial pivotal quantity (GFPQ) for parameter $\theta$, denoted as $G_{\theta}\left(S, S^{*}, \xi\right)$, is a function of $\left(S, S^{*}, \xi\right)$, and satisfies the following conditions:

(i) The conditional distribution of $G_{\theta}\left(S, S^{*}, \xi\right)$, given $S=s$, is free of $\xi$. 
(ii) For every allowable $s \in \mathcal{R}^{k}, G_{\theta}(s, s, \xi)=\theta$.

Approximate percentiles of $G_{\theta}\left(S, S^{*}, \xi\right)$ form a $100(1-\alpha) \%$ generalized fiducial CI for $\theta$. To wit, if the above conditions hold and there exist two statistics $L(s)$ and $U(s)$ such that

$$
\operatorname{Pr}\left\{L(s) \leq G_{\theta}\left(S, S^{*}, \xi\right) \leq U(s) \mid S=s\right\}=1-\alpha,
$$

thus the interval $(L(s), U(s))$ is called the $100(1-\alpha) \%$ generalized fiducial $\mathrm{CI}$ for $\theta$. In what follows, we adapt the above definition of generalized fiducial CI to determine NI margin via a historical placebo-controlled trial for the reference treatment, which is a superiority trial for establishing the efficacy of the reference treatment.

Let $Y_{i j}^{h}$ be the endpoint of the $j$ th individual for the $i$ th treatment in the historical trial for $i=R, P$ and $j=1, \ldots, m_{i}$. Following the aforementioned one-way fixed effect model, we assume that $Y_{P j}^{h}$ and $Y_{R j}^{h}$ follow the normal distributions, i.e.,

$$
\begin{aligned}
Y_{P j}^{h} \mid v_{P}, \xi_{P}^{2} & \sim \mathcal{N}\left(v_{P}, \xi_{P}^{2}\right), \quad j=1, \ldots, m_{P}, \\
Y_{R j}^{h} \mid v_{R}, \xi_{R}^{2} & \sim \mathcal{N}\left(v_{R}, \xi_{R}^{2}\right), \quad j=1, \ldots, m_{R},
\end{aligned}
$$

where $v_{P}$ and $v_{R}$ denote the treatment effects for placebo and reference treatments in the considered historical trial, respectively, $\xi_{P}^{2}$ and $\xi_{R}^{2}$ denote their corresponding variances to be estimated, and $m_{P}$ and $m_{R}$ are their corresponding sample sizes. Our purpose is to construct GFPQ for mean difference $\eta=v_{R}-v_{P}$. To this end, we denote $\bar{Y}_{k}^{h}=m_{k}^{-1} \sum_{j=1}^{m_{k}} Y_{k j}^{h}$ and $S_{k h}^{2}=\left(m_{k}-1\right)^{-1} \sum_{j=1}^{m_{k}}\left(Y_{k j}^{h}-\bar{Y}_{k}^{h}\right)^{2}$ for $k=R, P$. It is easily shown that $\hat{v}_{k}=\bar{Y}_{k}^{h}$ and $\hat{\xi}_{k}^{2}=S_{k h}^{2}$ are unbaised estimators of $v_{k}$ and $\xi_{k}^{2}$, respectively. Thus, an unbaised estimator of $\eta$ is given by $\hat{\eta}=\hat{v}_{R}-\hat{v}_{P}=\bar{Y}_{R}^{h}-\bar{Y}_{P}^{h}$. It is well known that $\bar{Y}_{k}^{h}$ and $S_{k h}^{2}$ are sufficient statstics of $v_{k}$ and $\xi_{k}^{2}$, respectively, and $\bar{Y}_{k}^{h} \sim \mathcal{N}\left(v_{k}, \xi_{k}^{2} / m_{k}\right)$ and $\left(m_{k}-1\right) S_{k h}^{2} \sim \xi_{k}^{2} \chi^{2}\left(m_{k}-1\right)$ for $k=R, P$. Let

$$
W_{k}^{h}=\frac{\bar{Y}_{k}^{h *}-v_{k}}{\xi_{k} / \sqrt{m_{k}}}, V_{k}^{h}=\frac{\left(m_{k}-1\right) S_{k h}^{2 *}}{\xi_{k}^{2}}
$$

where $\bar{Y}_{k}^{h *}$ and $S_{k h}^{2 *}$ are independent copies of $\bar{Y}_{k}^{h}$ and $S_{k h}^{2}$, respectively. Then, we have $W_{k}^{h} \sim \mathcal{N}(0,1), V_{k}^{h} \sim \chi^{2}\left(m_{k}-1\right)$, and $W_{k}^{h}$ is independent of $V_{k}^{h}$. Hence, the GFPQs for $v_{k}$ and $\xi_{k}^{2}$ are

$$
\begin{gathered}
R_{v_{k}}=\bar{Y}_{k}^{h}-\sqrt{\frac{S_{k h}^{2}}{S_{k h}^{2 *}}}\left(\bar{Y}_{k}^{h *}-v_{k}\right)=\bar{Y}_{k}^{h}-W_{k}^{h} \sqrt{\frac{\left(m_{k}-1\right) S_{k h}^{2}}{m_{k} V_{k}^{h}}}, \\
R_{\xi_{k}^{2}}=\frac{S_{k h}^{2}}{S_{k h}^{2 *}} \xi_{k}^{2}=\frac{\left(m_{k}-1\right) S_{k h}^{2}}{V_{k}^{h}},
\end{gathered}
$$

respectively. Using the GFPQs of $v_{R}$ and $v_{P}$ leads to

$$
\begin{aligned}
R_{\eta} & =\left(\bar{Y}_{R}^{h}-\bar{Y}_{P}^{h}\right)-\left(W_{R}^{h} \sqrt{\frac{\left(m_{R}-1\right) S_{R h}^{2}}{m_{R} V_{R}^{h}}}-W_{P}^{h} \sqrt{\frac{\left(m_{P}-1\right) S_{P h}^{2}}{m_{P} V_{P}^{h}}}\right) \\
& =\left(\bar{Y}_{R}^{h}-\bar{Y}_{P}^{h}\right)-\left(\sqrt{\frac{S_{R h}^{2}}{m_{R}}} \frac{W_{R}^{h}}{\sqrt{V_{R}^{h} /\left(m_{R}-1\right)}}-\sqrt{\frac{S_{P h}^{2}}{m_{P}}} \frac{W_{P}^{h}}{\sqrt{V_{P}^{h} /\left(m_{P}-1\right)}}\right) \\
& \triangleq\left(\bar{Y}_{R}^{h}-\bar{Y}_{P}^{h}\right)-\left(\sqrt{\frac{S_{R h}^{2}}{m_{R}}} T_{m_{R}-1}-\sqrt{\frac{S_{P h}^{2}}{m_{P}}} T_{m_{P}-1}\right),
\end{aligned}
$$


where $T_{m_{R}-1} \sim t\left(m_{R}-1\right)$ and $T_{m_{P}-1} \sim t\left(m_{P}-1\right)$. From Equation (10), it is easily shown that $R_{\eta}$ is a GFPQ of $\eta$. The $100(1-\alpha) \%$ two-sided GFCI for $\eta$ is $\left(R_{\eta, \alpha / 2}, R_{\eta, 1-\alpha / 2}\right)$, where $R_{\eta, \gamma}$ is the $100 \gamma \%$ percentile point of sample observations of $R_{\eta}$.

Denote $\Delta_{h}=R_{\eta, \alpha / 2}$. Following the frequentist's approach, we take the NI margin as $\Delta_{\mathrm{NI}}=(1-\lambda) \Delta_{h}$, where $\lambda \in[0,1]$ is a fraction representing the preservation level or desired proportion of the reference effect to the retained. Thus, the assay sensitivity margin can be expressed as $\Delta=\Delta_{\mathrm{NI}} / r=(1-\lambda) \Delta_{h} / r$. This shows that when $r<1-\lambda$ (i.e., $\Delta>\Delta_{h}>\Delta_{\mathrm{NI}}$ ), the assay sensitivity margin results in a more stringent rejection criterion than the historical placebo-controlled trial; when $r=1-\lambda$ and $\lambda \neq 0$ (i.e., $\Delta=\Delta_{h}>\Delta_{\mathrm{NI}}$ ), the assay sensitivity margin is equal to $\Delta_{h}$ and the NI margin is set as $(1-\lambda) \Delta_{h}$; when $r=1$ and $\lambda=0$, the hypothesis (3) reduces to the HT hypothesis.

\subsection{Simultaneous CIs for $\phi$ and $\psi$}

\subsubsection{Wald-Type Bonferroni Simultaneous CI}

Generally, to construct simultaneous CI for $\phi$ and $\psi$, one can consider the widely used and simple Bonferroni method based on the Wald-type statistics.

For the current trial data $X=\left\{X_{i j}: i=P, R, E, j=1, \ldots, n_{i}\right\}$ generated from model (2), we denote $\bar{X}_{i}=n_{i}^{-1} \sum_{j=1}^{n_{i}} X_{i j}$ and $S_{i}^{2}=\left(n_{i}-1\right)^{-1} \sum_{j=1}^{n_{i}}\left(X_{i j}-\bar{X}_{i}\right)^{2}$. The unbiased estimators of $\mu_{i}$ and $\sigma_{i}^{2}$ are given by $\hat{\mu}_{i}=\bar{X}_{i}$ and $\hat{\sigma}_{i}^{2}=S_{i}^{2}$, respectively, i.e., $E\left(\hat{\mu}_{i}\right)=\mu_{i}$ and $E\left(\hat{\sigma}_{i}^{2}\right)=\sigma_{i}^{2}$ for $i=P, R, E$. The unbiased estimators of $\phi$ and $\psi$ are $\hat{\phi}=\bar{X}_{E}-$ $\bar{X}_{R}$ and $\hat{\psi}=\bar{X}_{R}-\bar{X}_{P}$, respectively, whose variances are $\operatorname{var}(\hat{\phi})=\sigma_{E}^{2} / n_{E}+\sigma_{R}^{2} / n_{R}$ and $\operatorname{var}(\hat{\psi})=\sigma_{R}^{2} / n_{R}+\sigma_{P}^{2} / n_{P}$, respectively. The estimated variances of $\hat{\phi}$ and $\hat{\psi}$ have the forms: $\operatorname{vâr}(\hat{\phi})=\hat{\sigma}_{E}^{2} / n_{E}+\hat{\sigma}_{R}^{2} / n_{R}$ and $\operatorname{vâr}(\hat{\psi})=\hat{\sigma}_{R}^{2} / n_{R}+\hat{\sigma}_{P}^{2} / n_{P}$, respectively. It is easily shown that the Wald-type statistics $T_{\phi}=(\hat{\phi}-\phi) / \sqrt{\operatorname{varr}(\hat{\phi})}$ and $T_{\psi}=(\hat{\psi}-\psi) / \sqrt{\operatorname{vâr}(\hat{\psi})}$ asymptotically follow the standard normal distribution. Thus, an approximate $100(1-\alpha) \%$ one-sided Wald-type Bonferroni simultaneous confidence region for $\phi$ and $\psi$ is given by

$$
\left\{(\phi, \psi): \phi \in\left(L_{\phi}^{W B}, \infty\right), \psi \in\left(L_{\psi}^{W B}, \infty\right)\right\},
$$

where $L_{\phi}^{W B}=\hat{\phi}-z_{1-\alpha / 2} \sqrt{\operatorname{var}(\hat{\phi})}$, and $L_{\psi}^{W B}=\hat{\psi}-z_{1-\alpha / 2} \sqrt{\operatorname{var}(\hat{\psi})}$. The above defined simultaneous confidence region is called the WB-SCI.

As Tang et al. [13] pointed out, the Bonferroni simultaneous confidence region may behave poorly when $\min \left\{n_{E}, n_{R}, n_{P}\right\}$ is small. To address the issue, three new simultaneous confidence regions are developed as follows.

\subsubsection{Generalized Fiducial Simultaneous CI}

For $k=E, P, R$, let $\bar{X}_{k}^{*}$ and $S_{k}^{2 *}$ be independent copies of $\bar{X}_{k}$ and $S_{k}^{2}$, respectively, and define $W_{k}=\sqrt{n_{k}}\left(\bar{X}_{k}^{*}-\mu_{k}\right) / \sigma_{k}$ and $V_{k}=\left(n_{k}-1\right) S_{k}^{2 *} / \sigma_{k}^{2}$. It is easily shown that $W_{k} \sim \mathcal{N}(0,1), V_{k} \sim \chi^{2}\left(n_{k}-1\right)$ and $W_{k}$ is independent of $V_{k}$. It follows from Section 2.1 that the GFPQs for $\sigma_{k}^{2}$ and $\mu_{k}$ can be expressed as

$$
R_{\sigma_{k}^{2}}=\frac{\left(n_{k}-1\right) S_{k}^{2}}{V_{k}}, R_{\mu_{k}}=\bar{X}_{k}-W_{k} \sqrt{\frac{\left(n_{k}-1\right) S_{k}^{2}}{n_{k} V_{k}}}
$$

respectively. Thus, the GFPQs of $\phi$ and $\psi$ are

$$
\begin{aligned}
& R_{\phi}=\bar{X}_{E}-\bar{X}_{R}-\left(W_{E} \sqrt{\frac{\left(n_{E}-1\right) S_{E}^{2}}{n_{E} V_{E}}}-W_{R} \sqrt{\frac{\left(n_{R}-1\right) S_{R}^{2}}{n_{R} V_{R}}}\right), \\
& R_{\psi}=\bar{X}_{R}-\bar{X}_{P}-\left(W_{R} \sqrt{\frac{\left(n_{R}-1\right) S_{R}^{2}}{n_{R} V_{R}}}-W_{P} \sqrt{\frac{\left(n_{P}-1\right) S_{P}^{2}}{n_{P} V_{P}}}\right),
\end{aligned}
$$


respectively. Define

$$
R_{D}=\max \left(\frac{\hat{\phi}-R_{\phi}}{\sqrt{\operatorname{vâr}(\hat{\phi})}}, \frac{\hat{\psi}-R_{\psi}}{\sqrt{\operatorname{vâr}(\hat{\psi})}}\right) .
$$

Thus, the $100(1-\alpha) \%$ one-sided simultaneous generalized fiducial confidence region for $(\phi, \psi)$ is

$$
\left\{(\phi, \psi): \phi \in\left(L_{\phi}^{F}, \infty\right), \psi \in\left(L_{\psi}^{F}, \infty\right)\right\}
$$

where $L_{\phi}^{F}=\hat{\phi}-d_{1-\alpha} \sqrt{\operatorname{vâr}(\hat{\phi})}, L_{\psi}^{F}=\hat{\psi}-d_{1-\alpha} \sqrt{\operatorname{vâr}(\hat{\psi})}$, and $d_{1-\alpha}$ is the $1-\alpha$ percentile point of statistic $R_{D}$ 's distribution given sample observations. The above defined simultaneous confidence region is referred to as GF-SCI method.

Theorem 1. Let $X_{k 1}, \ldots, X_{k n_{k}}$ be an independent and identically distributed sample from $\mathcal{N}\left(\mu_{k}, \sigma_{k}^{2}\right)$ for $k=P, R$, E. Suppose that $r_{k}=\lim _{n \rightarrow \infty} n_{k} / n$ holds, where $n=n_{E}+n_{R}+n_{P}$ and $0<r_{k}<1$ for $k=P, R, E$. Then, we have

$$
\operatorname{Pr}\left(\phi \geq L_{\phi}^{F}, \psi \geq L_{\psi}^{F}\right) \approx 1-\alpha
$$

The proof of Theorem 1 is given in Appendix A. Theorem 1 shows that the proposed GF-SCI can asymptotically attain the pre-specified coverage probability.

Combining the above argument, we form the following computing Algorithm 1 for evaluating empirical coverage probability (ECP) of the proposed GF-SCI.

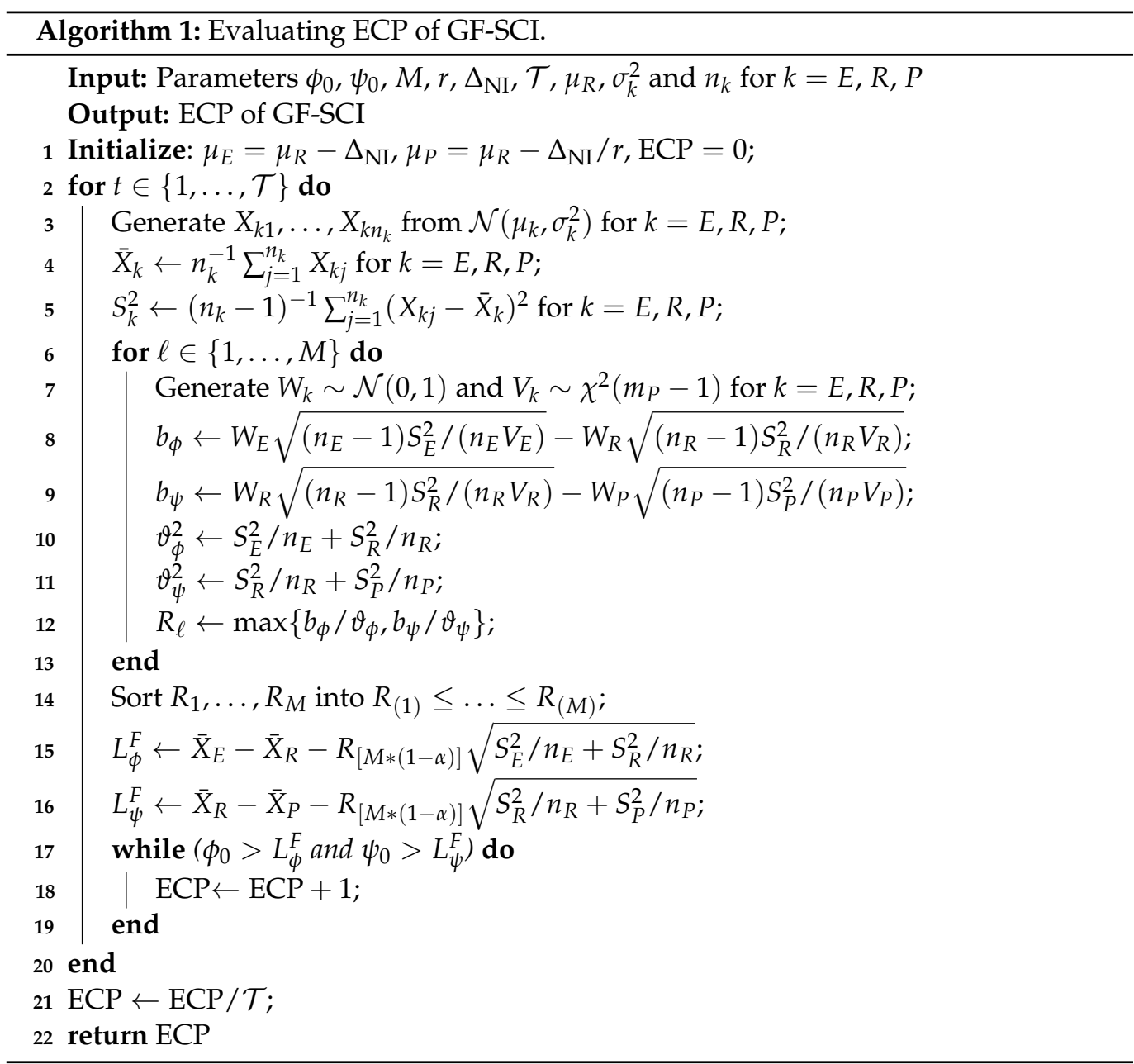




\subsubsection{Hybrid Generalized Fiducial Simultaneous CI}

The above presented GF-SCI for $\phi$ and $\psi$ is computationally intensive in determining the percentile point of statistic $R_{D}$ 's distribution. To solve this problem, we develop a hybrid generalized fiducial simultaneous $\mathrm{CI}$ by incorporating the square-and-add method and generalized fiducial method as follows.

The lower limits of the approximate $100(1-\alpha) \%$ Wald-type CIs for $\phi$ and $\psi$ are given by

$$
L_{\phi}^{W}=\hat{\phi}-z_{1-\alpha} \sqrt{\operatorname{var}(\hat{\phi})}, \quad L_{\psi}^{W}=\hat{\psi}-z_{1-\alpha} \sqrt{\operatorname{vâr}(\hat{\psi})},
$$

respectively, where $\operatorname{vâr}(\hat{\phi})=\operatorname{vâr}\left(\hat{\mu}_{E}\right)+\operatorname{vâr}\left(\hat{\mu}_{R}\right)$, $\operatorname{vâr}(\hat{\psi})=\operatorname{vâr}\left(\hat{\mu}_{R}\right)+\operatorname{vâr}\left(\hat{\mu}_{P}\right)$, and $z_{1-\alpha}$ is the $1-\alpha$ percentile point of the standard normal distribution. It is easily shown that vâr $(\hat{\phi})$ and $\operatorname{vâr}(\hat{\psi})$ are consistent estimators of $\operatorname{var}(\hat{\phi})$ and $\operatorname{var}(\hat{\psi})$ when the sample sizes $n_{R}$ and $n_{P}$ are sufficiently large, respectively. Hence, to recovery variances $\operatorname{var}(\hat{\phi})$ and $\operatorname{var}(\hat{\psi})$ so that the resultant simultaneous CI behaves satisfactorily for small to moderate sample sizes, we develop the following hybrid method, which is similar to that given in [14].

Let $l_{k}$ and $u_{k}$ be the lower and upper limits of an approximate $100(1-\alpha) \%$ one-sided CI for $\mu_{k}$ for $k=E, R, P$. It follows from the Slutsky's theorem that statistic $T_{k}=\left(\hat{\mu}_{k}-\right.$ $\left.\mu_{k}\right) / \sqrt{\operatorname{vâr}\left(\hat{\mu}_{k}\right)}$ asymptotically follows the standard normal distribution for $k=E, R, P$. Thus, for $k=E, R, P$, we have $l_{k}=\hat{\mu}_{k}-z_{1-\alpha} \sqrt{\operatorname{vâr}\left(\hat{\mu}_{k}\right)}$ and $u_{k}=\hat{\mu}_{k}+z_{1-\alpha} \sqrt{\operatorname{vâr}\left(\hat{\mu}_{k}\right)}$, which lead to $\operatorname{vâr}\left(\hat{\mu}_{k}\right)=\left(\hat{\mu}_{k}-l_{k}\right)^{2} / Z_{1-\alpha}^{2}$ for $l_{k}$ and $\operatorname{vâr}\left(\hat{\mu}_{k}\right)=\left(u_{k}-\hat{\mu}_{k}\right)^{2} / Z_{1-\alpha}^{2}$ for $u_{k}$, respectively. Following the argument of Howe [23] and Newcombe [11], we substitute the above equations into (18) and define the lower limits of the approximate $100(1-\alpha) \%$ one-sided hybrid CIs for $\phi$ and $\psi$ as

$$
L_{\phi}^{H}=\hat{\phi}-\sqrt{\left(\hat{\mu}_{E}-l_{E}\right)^{2}+\left(u_{R}-\hat{\mu}_{R}\right)^{2}}, \quad L_{\psi}^{H}=\hat{\psi}-\sqrt{\left(\hat{\mu}_{R}-l_{R}\right)^{2}+\left(u_{P}-\hat{\mu}_{P}\right)^{2}},
$$

respectively, which imply that it is necessary to first evaluate the lower and upper limits $l_{k}$ and $u_{k}(k=E, R, P)$ for computing $L_{\phi}^{H}$ and $L_{\psi}^{H}$. In what follows, the generalized fiducial method introduced above is employed to calculate $l_{k}$ and $u_{k}$ for $k=E, R, P$.

The lower and upper limits of an approximate 100(1- $\alpha) \%$ one-sided GFCI of $\mu_{k}$ adjusted for multiplicity using the Bonferroni method have the forms

$$
l_{k}=R_{\mu_{k}, \alpha / 2}, \quad u_{k}=R_{\mu_{k}, 1-\alpha / 2},
$$

where $R_{\mu_{k}, \alpha}$ represents the $\alpha$ percentile point of sample observations of $R_{\mu_{k}}$, which is the GFPQ of $\mu_{k}$ with $R_{\mu_{k}}=\bar{X}_{k}-W_{k} \sqrt{\left(n_{k}-1\right) S_{k}^{2} /\left(n_{k} V_{k}\right)}, W_{k} \sim \mathcal{N}(0,1)$ and $V_{k} \sim \chi^{2}\left(n_{k}-1\right)$ for $k=E, R, P$.

Plugging the above defined lower and upper limits $l_{k}$ and $u_{k}(k=E, R, P)$ into $L_{\phi}^{H}$ and $L_{\psi}^{H}$ defined in (19) leads to an approximate $100(1-\alpha) \%$ one-sided hybrid generalized fiducial simultaneous confidence region of $(\phi, \psi)$ :

$$
\left\{(\phi, \psi): \phi \in\left(L_{\phi}^{H}, \infty\right), \psi \in\left(L_{\psi}^{H}, \infty\right)\right\} .
$$

The above defined simultaneous confidence region is referred to as HG-SCI method.

The approach to evaluate empirical coverage probability (ECP) of the defined HG-SCI is summarized as the Algorithm 2. 


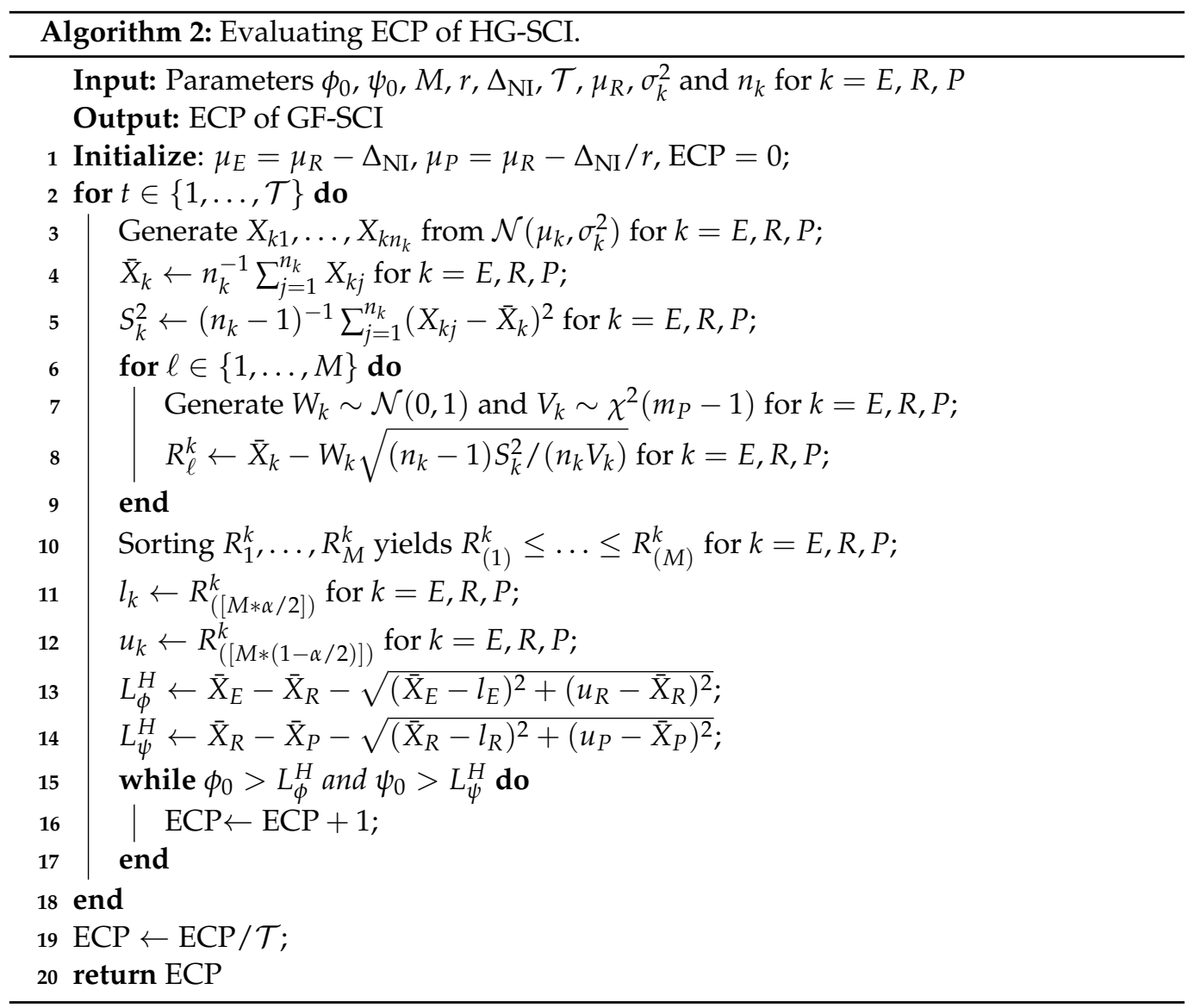

\subsubsection{Parametric Bootstrap Simultaneous CI}

From Section 2.2.3, it is easily seen that the current data are repeatedly used in calculating hybrid generalized fiducial simultaneous confidence region. To address the issue, following [22], we consider the following parametric bootstrap simultaneous confidence region for $\phi$ and $\psi$.

To this end, we denote

$$
T_{\phi}=\frac{\hat{\phi}-\phi}{\sqrt{\operatorname{vâr}(\hat{\phi})}}, T_{\psi}=\frac{\hat{\psi}-\psi}{\sqrt{\operatorname{vâr}(\hat{\psi})}}, T_{B}=\max \left(T_{\phi}, T_{\psi}\right) .
$$

Let $q_{1-\alpha}$ be the approximate $1-\alpha$ percentile point of statistic $T_{B}$ 's distribution. Then, the lower limits of an approximate $100(1-\alpha) \%$ one-sided simultaneous confidence region for $\phi$ and $\psi$ are given as

$$
L_{\phi}^{B}=\hat{\phi}-q_{1-\alpha} \sqrt{\operatorname{vâr}(\hat{\phi})}, \quad L_{\psi}^{B}=\hat{\psi}-q_{1-\alpha} \sqrt{\operatorname{vâr}(\hat{\psi})},
$$


respectively. Clearly, the challenge for evaluating $L_{\phi}^{B}$ and $L_{\psi}^{B}$ is to calculate $q_{1-\alpha}$. While $T_{\phi}$ and $T_{\psi}$ are asymptotically distributed as the standard normal distribution, it is rather difficult to compute $q_{1-\alpha}$ from the joint distribution of $T_{\phi}$ and $T_{\psi}$ in that $T_{\phi}$ is not independent of $T_{\psi}$. To solve the problem, the parametric bootstrap approach given in [22] is adopted to approximate the distribution of statistic $T_{B}$.

Let $s_{k}^{2}$ denote the observed values of $S_{k}^{2}$ for $k=E, P, R$. Note that the distribution of $T_{B}$ depends on the nuisance parameters $\sigma_{k}^{2}$, but it dose not depend on the values of $\mu_{k}$ for $k=E, R, P$. Therefore, when inducing the distribution of $T_{B}$, we can take $\mu_{k}=0$ for $k=E$, $R, P$. The parametric bootstrap distribution of $T_{B}$ can be obtained from its resampling distribution when the values of $\sigma_{k}^{2}$ 's are replaced by $s_{k}^{2}$ and the values of $\mu_{k}$ 's are taken as zero. It follows from Equation (22) that the parametric bootstrap pivotal quantity for statistic $T_{B}$ is

$$
T_{B}^{P I}=\max \left(\frac{\bar{X}_{E}^{B}-\bar{X}_{R}^{B}}{\sqrt{\operatorname{vâr}_{B}(\hat{\phi})}}, \frac{\bar{X}_{R}^{B}-\bar{X}_{P}^{B}}{\sqrt{\operatorname{vâr}_{B}(\hat{\psi})}}\right),
$$

where

$$
\begin{gathered}
\operatorname{vâr}_{B}(\hat{\phi})=\frac{S_{E, B}^{2}}{n_{E}}+\frac{S_{R, B}^{2}}{n_{R}}, \quad \operatorname{vâr}_{B}(\hat{\psi})=\frac{S_{R, B}^{2}}{n_{R}}+\frac{S_{P, B}^{2}}{n_{P}}, \\
\bar{X}_{k}^{B} \sim \mathcal{N}\left(0, \frac{s_{k}^{2}}{n_{k}}\right), \quad S_{k, B}^{2} \sim \frac{s_{k}^{2}}{n_{k}-1} \chi^{2}\left(n_{k}-1\right) .
\end{gathered}
$$

The distribution of $T_{B}^{P I}$ provides a parametric bootstrap approximation to the distribution of $T_{B}$. Let $q_{1-\alpha}^{B}$ be the $1-\alpha$ percentile point of bootstrap sample observations of $T_{B}^{P I}$. Thus, an approximate $100(1-\alpha) \%$ one-sided parametric bootstrap simultaneous confidence region for $(\phi, \psi)$ is given by

$$
\left\{(\phi, \psi): \phi \in\left(L_{\phi}^{B}, \infty\right), \psi \in\left(L_{\psi}^{B}, \infty\right)\right\},
$$

where $L_{\phi}^{B}=\hat{\phi}-q_{1-\alpha}^{B} \sqrt{\operatorname{vâr}(\hat{\phi})}$, and $L_{\psi}^{B}=\hat{\psi}-q_{1-\alpha}^{B} \sqrt{\operatorname{vâr}(\hat{\psi})}$. The above defined simultaneous confidence region is referred to as PB-SCI method.

Theorem 2. Let $X_{k 1}, \ldots, X_{k n_{k}}$ be an independent and identically distributed sample from $\mathcal{N}\left(\mu_{k}, \sigma_{k}^{2}\right)$ for $k=P, R, E$. Let $n=n_{E}+n_{R}+n_{P}$. Assume that there is a $r_{k} \in(0,1)$ such that $r_{k}=\lim _{n \rightarrow \infty} n_{k} / n$ holds for $k=E, R, P$. Then, we have

$$
\operatorname{Pr}\left(\phi \geq L_{\phi}^{B}, \psi \geq L_{\psi}^{B}\right) \approx 1-\alpha .
$$

Proof of Theorem 2 is given in Appendix B. Theorem 2 shows that the preceding proposed parametric bootstrap simultaneous confidence region can asymptotically attain the pre-specified confidence level $1-\alpha$. 


\subsection{Sample Size Determination}

Due to the duality of hypothesis testing and interval estimation, we can obtain the rejection regions at the significance level $\alpha=0.05$ from the above proposed four simultaneous confidence regions, which are given as $\mathbb{D}_{W}=\left\{\left(x_{E}, x_{R}, x_{P}\right): \bar{x}_{E}-\bar{x}_{R}>\right.$ $\left.-\hat{\Delta}_{\mathrm{NI}}+z_{1-\alpha / 2} \sqrt{\operatorname{vâr}(\hat{\phi})} \cup \bar{x}_{R}-\bar{x}_{P}>\hat{\Delta}+z_{1-\alpha / 2} \sqrt{\operatorname{vâr}(\hat{\psi})}\right\}, \mathbb{D}_{F}=\left\{\left(x_{E}, x_{R}, x_{P}\right): \bar{x}_{E}-\bar{x}_{R}>\right.$ $\left.-\hat{\Delta}_{\mathrm{NI}}+d_{1-\alpha} \sqrt{\operatorname{vâr}(\hat{\phi})} \cup \bar{x}_{R}-\bar{x}_{P}>\hat{\Delta}+d_{1-\alpha} \sqrt{\operatorname{vâr}(\hat{\psi})}\right\}, \mathbb{D}_{H}=\left\{\left(x_{E}, x_{R}, x_{P}\right): \bar{x}_{E}-\bar{x}_{R}>\right.$ $\left.-\hat{\Delta}_{\mathrm{NI}}+\sqrt{\left(\hat{\mu}_{E}-l_{E}\right)^{2}+\left(u_{R}-\hat{\mu}_{R}\right)^{2}} \cup \bar{x}_{R}-\bar{x}_{P}>\hat{\Delta}+\sqrt{\left(\hat{\mu}_{R}-l_{R}\right)^{2}+\left(u_{P}-\hat{\mu}_{P}\right)^{2}}\right\}, \mathbb{D}_{B}=$ $\left\{\left(x_{E}, x_{R}, x_{P}\right): \bar{x}_{E}-\bar{x}_{R}>-\hat{\Delta}_{\mathrm{NI}}+q_{1-\alpha}^{B} \sqrt{\operatorname{vâr}(\hat{\phi})} \cup \bar{x}_{R}-\bar{x}_{P}>\hat{\Delta}+q_{1-\alpha}^{B} \sqrt{\operatorname{vâr}(\hat{\psi})}\right\}, \operatorname{respec}-$ tively, where $\bar{x}_{k}$ denotes the sample mean for $k=E, R, P$, and $\mathbb{D}_{W}, \mathbb{D}_{F}, \mathbb{D}_{H}$ and $\mathbb{D}_{B}$ correspond to the WB-SCI, GF-SCI, HG-SCI and PB-SCI, respectively. The family wise error rate can be controlled at the significance level $\alpha$ via $\operatorname{Pr}\left(\mathbb{D}_{\ell} \mid H_{\mathrm{NI}}^{0}, K_{\mathrm{AS}}^{0}\right) \approx \alpha$, and the power of $1-\beta$ can be computed by $\operatorname{Pr}\left(\mathbb{D}_{\ell} \mid H_{\mathrm{NI}}^{1}, K_{\mathrm{AS}}^{1}\right) \approx 1-\beta$ for $\ell=\mathrm{W}, \mathrm{F}, \mathrm{H}$ and $\mathrm{B}$. The required sample size $n=n_{E}+n_{R}+n_{P}$ for attaining the pre-specified power $1-\beta$ at the given significance level $\alpha$ can be obtained by finding the solution to $\operatorname{Pr}\left(\mathbb{D}_{\ell} \mid H_{\mathrm{NI}}^{1}, K_{\mathrm{AS}}^{1}\right) \approx 1-\beta$ with respect to $n$ for $\ell=\mathrm{W}, \mathrm{F}, \mathrm{H}$ and $\mathrm{B}$. There is not a closed-form for $n$. To this end, Algorithm 3 is presented to compute the sample size $n$ with the assumption: $n_{E}: n_{R}: n_{P}=c_{1}: c_{2}: 1$ as follows.

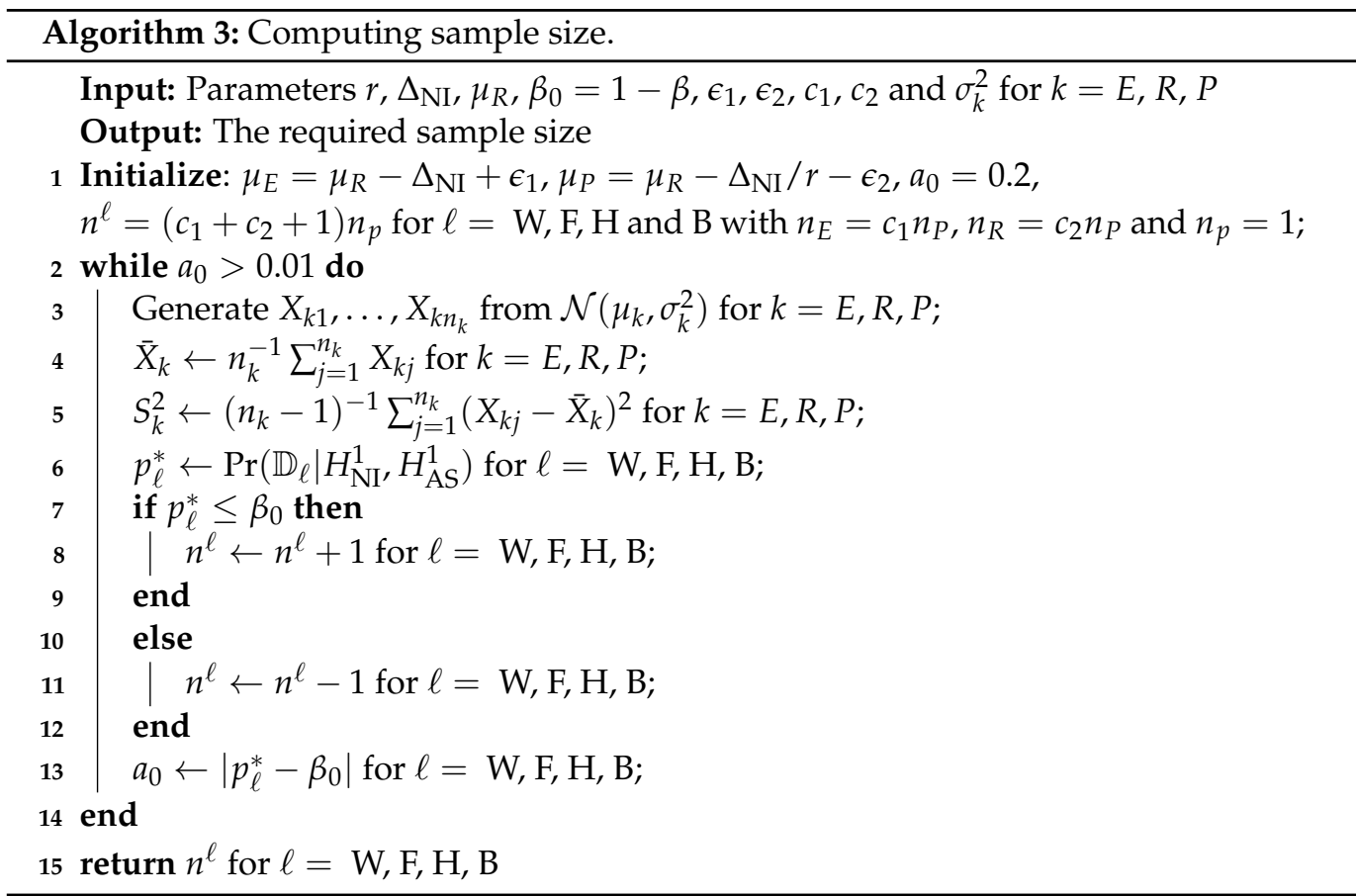

\section{Results}

Simulation examples and a real example are provided to illustrate the efficiency of the preceding proposed methods in terms of empirical coverage probabilities. The lower limits of the approximate $95 \%$ one-sided simultaneous confidence region for the proposed methods are given in an application to mildly asthmatic study. Empirical results demonstrated that the GF-SCI and HG-SCI perform better than the WB-SCI and PB-SCI.

\subsection{Simulation Results}

In this section, two simulation studies are conducted to investigate the performance of the preceding proposed methodologies. 
To compare the performance of the proposed simultaneous confidence regions in terms of their empirical coverage probabilities (ECPs), we conduct the first simulation study for $\mathcal{T}=5000$ replications. In this simulation study, we take $v_{R}=4, v_{P}=1.5, \xi_{R}^{2}=1$, $\xi_{P}^{2}=2$ and $m_{P}=m_{R}=20$ in generating historical data for determining $\Delta_{N I}$ and $\Delta=\Delta_{\mathrm{NI}} / r$ with $M=5000$, and $\sigma_{P}^{2}=2.0, \sigma_{E}^{2}=1.5, \sigma_{R}^{2}=0.5,1.0$ and 3.0, $\mu_{R}=3.0,4.0$ and 5.0, $\mu_{E}=\mu_{R}-\Delta_{\mathrm{NI}}$ and $\mu_{P}=\mu_{R}-\Delta$ indicating that the true values $\phi_{0}$ and $\psi_{0}$ of $\phi$ and $\psi$ are $\phi_{0}=-\Delta_{\mathrm{NI}}$ and $\psi_{0}=\Delta$, respectively, in sampling the current trial data. We set $r=0.3$ together with $\lambda=0.6$ and 0.7 , and $r=0.6$ together with $\lambda=0.3$ and 0.4 , corresponding to the cases that $\Delta>\Delta_{h}>\Delta_{\mathrm{NI}}$ and $\Delta=\Delta_{h}>\Delta_{\mathrm{NI}}$, respectively; and $r=1.0$ together with $\lambda=0.0$ corresponding to the case that $\Delta=\Delta_{h}=\Delta_{\mathrm{NI}}$. We consider $n=n_{E}+n_{R}+n_{P}=60$, 150 and 300 with the following three allocation proportions: (L1) the balanced design $n_{E}: n_{R}: n_{P}=1: 1: 1$, (L2) the unbalanced design $n_{E}: n_{R}: n_{P}=2: 2: 1$ and (L3) the unbalanced design $n_{E}: n_{R}: n_{P}=3: 2: 1$.

For each of the aforementioned settings, the preceding proposed three algorithms for evaluating simultaneous confidence regions for $\phi$ and $\psi$ are used to compute ECPs at the significance level $\alpha=0.05$. Results for $n=60,150$ and 300 are given in Tables $1-3$, respectively. Inspection of Tables 1-3 indicates that (i) the GF-SCI and HG-SCI methods behave better than the WB-SCI and PB-SCI methods regardless of the values of $r, \lambda, \mu_{R}$ and $\sigma_{R}^{2}$ and the sample sizes in that the ECPs of the former are quite closer to the pre-specified confidence level 95\% than those of the latter; (ii) when the sample size $n$ is small, the PB-SCI and WB-SCI methods are liberal regardless of the balanced and unbalanced designs in that their ECPs are less than the pre-specified confidence level 95\%, but the PB-SCI method behaves better than the WB-SCI method in that the ECPs for the former are closer to the pre-specified confidence level than those for the latter; (iii) when the sample size is large (e.g., $n=150$ and 300), the PB-SCI method has the same performance as the GF-SCI and HG-SCI methods, but the WB-SCI method is liberal.

To investigate the performance of the sample size determination method introduced above (i.e., Algorithm 3), we conduct the second simulation study. Here the same settings as those given in the first simulation study including $r$ and $\lambda$ are used to generate the historical data in determining the NI margin: $\Delta_{\mathrm{NI}}=(1-\lambda) \Delta_{h}$ and $\Delta=(1-\lambda) \Delta_{h} / r$, i.e., $v_{R}=4, v_{P}=1.5, \xi_{R}^{2}=1, \xi_{P}^{2}=2, m_{P}=m_{R}=20, M=5000, r=0.3$ and $\lambda=0.6$ and 0.7, $r=0.6$ and $\lambda=0.3$ and 0.4 , and $r=1.0$ and $\lambda=0.0$. To calculate the required sample size for attaining the power $\beta_{0}=1-\beta=0.95$, we consider the following settings: $\sigma_{P}^{2}=2.0$, $\sigma_{E}^{2}=1.5, \sigma_{R}^{2}=0.5,1.0$ and 3.0, $\mu_{R}=3,4$ and $5, \mu_{E}=\mu_{R}-\Delta_{\mathrm{NI}}+\epsilon_{1}, \mu_{P}=\mu_{R}-\Delta-\epsilon_{2}$ with $2 \epsilon_{1}=\epsilon_{2}=0.4$ and 0.6 , and four allocation proportions: $n_{E}: n_{R}: n_{P}=1: 1: 1$ (the balanced design), and 2:1:1, 2:2:1 and 3:2:1 (the unbalanced design).

For each of the aforementioned settings, the above introduced Algorithm 3 is adopted to calculate the required sample sizes for the WB-SCI, GF-SCI, HG-SCI and PB-SCI methods. The powers $\operatorname{Pr}\left(\mathbb{D}_{\ell} \mid H_{\mathrm{NI}}^{1}, H_{\mathrm{AS}}^{1}\right)$ for $\ell=\mathrm{W}, \mathrm{F}, \mathrm{H}$ and $\mathrm{B}$ are computed by Monte Carlo method with $\mathcal{T}=5000$ random observations. Based on the required sample size, we also compute its corresponding empirical power for comparing the accuracy of the proposed sample size determination. Results for $\alpha=0.05$ are presented in Tables 4 and 5. Examination of Tables 4 and 5 shows that (i) the derived sample size formulae are rather accurate regardless of the values of $r, \lambda$ and $\epsilon_{2}$, the balanced and unbalanced designs in that their corresponding empirical powers are quite close to the pre-specified true powers; (ii) the sample size increases as $\sigma_{R}^{2}$ increases; (iii) the value of $\mu_{R}$ has little effect on the sample size; (iv) the sample size decreases as $\epsilon_{2}$ increases. 
Table 1. Empirical coverage probabilities of four $95 \%$ simultaneous confidence regions for $\phi$ and $\psi$ under various settings with $n=60$.

\begin{tabular}{|c|c|c|c|c|c|c|c|c|c|c|c|c|c|c|c|}
\hline \multirow[b]{2}{*}{$r$} & \multirow[b]{2}{*}{$\lambda$} & \multirow[b]{2}{*}{$\mu_{R}$} & \multirow[b]{2}{*}{$\sigma_{R}^{2}$} & \multicolumn{4}{|c|}{$n_{E}: n_{R}: n_{P}=1: 1: 1$} & \multicolumn{4}{|c|}{$n_{E}: n_{R}: n_{P}=2: 2: 1$} & \multicolumn{4}{|c|}{$n_{E}: n_{R}: n_{P}=3: 2: 1$} \\
\hline & & & & GF & HG & WB & PB & GF & HG & WB & PB & GF & HG & WB & PB \\
\hline \multirow[t]{18}{*}{0.3} & \multirow[t]{9}{*}{0.6} & \multirow[t]{3}{*}{3} & 0.5 & 95.00 & 95.06 & 93.90 & 94.68 & 95.16 & 95.14 & 93.26 & 94.62 & 95.22 & 95.36 & 93.42 & 94.78 \\
\hline & & & 1 & 95.34 & 95.44 & 94.02 & 94.66 & 95.34 & 95.28 & 93.34 & 94.66 & 95.52 & 95.60 & 93.62 & 94.98 \\
\hline & & & 3 & 95.44 & 95.42 & 94.10 & 94.80 & 95.70 & 95.86 & 94.34 & 95.18 & 96.02 & 96.04 & 94.48 & 95.36 \\
\hline & & \multirow[t]{3}{*}{4} & 0.5 & 94.98 & 95.10 & 93.62 & 94.50 & 95.42 & 95.66 & 93.82 & 95.14 & 95.20 & 95.32 & 93.38 & 94.74 \\
\hline & & & 1 & 95.22 & 95.36 & 93.64 & 94.48 & 95.50 & 95.60 & 93.92 & 95.12 & 95.80 & 95.78 & 93.74 & 95.16 \\
\hline & & & 3 & 95.44 & 95.60 & 94.26 & 95.04 & 95.58 & 95.64 & 94.26 & 95.04 & 95.72 & 95.76 & 93.90 & 95.14 \\
\hline & & \multirow[t]{3}{*}{5} & 0.5 & 95.52 & 95.66 & 94.16 & 95.16 & 95.44 & 95.48 & 93.98 & 94.98 & 95.64 & 95.44 & 93.46 & 95.06 \\
\hline & & & 1 & 95.70 & 95.74 & 94.40 & 95.12 & 95.54 & 95.44 & 93.90 & 94.98 & 95.86 & 95.88 & 93.98 & 95.28 \\
\hline & & & 3 & 95.94 & 95.88 & 94.44 & 95.20 & 96.00 & 96.12 & 94.42 & 95.22 & 96.12 & 96.10 & 94.18 & 95.38 \\
\hline & \multirow[t]{9}{*}{0.7} & \multirow[t]{3}{*}{3} & 0.5 & 95.46 & 95.56 & 94.16 & 95.00 & 95.60 & 95.70 & 94.10 & 95.24 & 95.64 & 95.52 & 93.84 & 95.14 \\
\hline & & & 1 & 95.38 & 95.48 & 93.88 & 94.66 & 95.22 & 95.38 & 93.68 & 94.66 & 95.58 & 95.64 & 93.78 & 94.92 \\
\hline & & & 3 & 95.50 & 95.62 & 94.18 & 95.04 & 95.36 & 95.46 & 93.86 & 94.88 & 96.20 & 96.44 & 94.46 & 95.42 \\
\hline & & \multirow[t]{3}{*}{4} & 0.5 & 95.58 & 95.58 & 94.22 & 95.24 & 94.54 & 94.72 & 92.80 & 94.18 & 95.64 & 95.74 & 93.66 & 95.10 \\
\hline & & & 1 & 95.86 & 95.92 & 94.28 & 95.12 & 95.82 & 95.76 & 94.12 & 95.32 & 95.36 & 95.42 & 93.50 & 94.70 \\
\hline & & & 3 & 95.30 & 95.42 & 93.86 & 94.76 & 95.44 & 95.56 & 93.96 & 94.82 & 95.82 & 95.86 & 94.38 & 95.12 \\
\hline & & \multirow[t]{3}{*}{5} & 0.5 & 95.68 & 95.82 & 94.38 & 95.42 & 95.26 & 95.46 & 93.76 & 95.06 & 95.34 & 95.46 & 93.32 & 95.12 \\
\hline & & & 1 & 95.90 & 95.94 & 94.42 & 95.36 & 95.26 & 95.38 & 93.54 & 94.58 & 95.54 & 95.76 & 93.72 & 95.16 \\
\hline & & & 3 & 95.92 & 95.94 & 94.46 & 95.34 & 95.64 & 95.62 & 93.94 & 94.86 & 95.82 & 95.82 & 94.06 & 95.10 \\
\hline \multirow[t]{18}{*}{0.6} & \multirow[t]{9}{*}{0.3} & 3 & 0.5 & 94.80 & 94.96 & 93.48 & 94.32 & 94.84 & 95.00 & 93.26 & 94.42 & 95.12 & 95.16 & 93.16 & 94.44 \\
\hline & & & 1 & 95.34 & 95.48 & 94.20 & 94.74 & 95.62 & 95.82 & 93.96 & 95.10 & 95.22 & 95.28 & 93.36 & 94.68 \\
\hline & & & 3 & 95.62 & 95.64 & 94.24 & 95.10 & 96.08 & 96.26 & 94.76 & 95.54 & 95.40 & 95.46 & 93.40 & 94.90 \\
\hline & & 4 & 0.5 & 95.66 & 95.80 & 94.38 & 95.22 & 95.52 & 95.68 & 93.78 & 95.16 & 95.18 & 95.38 & 93.34 & 94.52 \\
\hline & & & 1 & 95.26 & 95.52 & 93.72 & 94.74 & 95.06 & 95.24 & 93.36 & 94.60 & 95.86 & 95.98 & 94.26 & 95.38 \\
\hline & & & 3 & 95.64 & 95.80 & 94.26 & 94.96 & 95.78 & 95.80 & 94.18 & 95.18 & 95.38 & 95.62 & 93.56 & 94.74 \\
\hline & & 5 & 0.5 & 94.60 & 94.70 & 93.44 & 94.18 & 94.90 & 95.16 & 93.46 & 94.62 & 95.04 & 95.00 & 92.86 & 94.42 \\
\hline & & & 1 & 95.32 & 95.36 & 93.88 & 94.76 & 95.54 & 95.52 & 93.72 & 94.72 & 95.94 & 96.04 & 93.98 & 95.44 \\
\hline & & & 3 & 95.18 & 95.26 & 93.68 & 94.60 & 95.66 & 95.74 & 94.38 & 95.10 & 95.66 & 95.58 & 93.80 & 94.94 \\
\hline & 0.4 & 3 & 0.5 & 94.84 & 94.92 & 93.56 & 94.42 & 95.68 & 95.74 & 94.08 & 95.28 & 95.48 & 95.58 & 93.48 & 95.02 \\
\hline & & & 1 & 95.14 & 95.36 & 93.94 & 94.78 & 95.28 & 95.28 & 94.12 & 94.96 & 95.22 & 95.36 & 93.34 & 94.46 \\
\hline & & & 3 & 95.92 & 95.94 & 94.70 & 95.52 & 95.84 & 95.84 & 94.44 & 95.18 & 95.60 & 95.60 & 93.86 & 95.10 \\
\hline & & 4 & 0.5 & 95.28 & 95.34 & 93.88 & 94.84 & 95.10 & 95.18 & 93.26 & 94.70 & 95.20 & 95.16 & 93.46 & 94.70 \\
\hline & & & 1 & 95.78 & 95.98 & 94.26 & 95.22 & 95.92 & 96.04 & 94.28 & 95.46 & 95.50 & 95.50 & 93.58 & 94.88 \\
\hline & & & 3 & 95.50 & 95.64 & 94.00 & 94.82 & 95.72 & 95.90 & 94.58 & 95.20 & 95.58 & 95.60 & 93.92 & 94.98 \\
\hline & & 5 & 0.5 & 95.82 & 95.84 & 94.42 & 95.34 & 95.38 & 95.48 & 93.84 & 95.00 & 95.28 & 95.30 & 93.40 & 94.72 \\
\hline & & & 1 & 94.56 & 94.66 & 93.56 & 94.08 & 95.66 & 95.78 & 94.00 & 95.00 & 95.16 & 95.36 & 93.44 & 94.76 \\
\hline & & & 3 & 96.02 & 96.04 & 94.52 & 95.38 & 95.08 & 95.26 & 93.82 & 94.44 & 95.48 & 95.32 & 93.82 & 94.58 \\
\hline 1.0 & 0.0 & 3 & 0.5 & 95.52 & 95.60 & 94.20 & 95.08 & 95.38 & 95.38 & 93.86 & 95.00 & 95.68 & 95.88 & 94.00 & 95.16 \\
\hline & & & 1 & 95.64 & 95.70 & 94.10 & 95.02 & 94.98 & 95.16 & 93.54 & 94.44 & 95.46 & 95.58 & 93.28 & 94.74 \\
\hline & & & 3 & 95.64 & 95.68 & 94.20 & 94.92 & 95.36 & 95.46 & 94.00 & 94.82 & 95.56 & 95.44 & 93.74 & 94.80 \\
\hline & & 4 & 0.5 & 95.32 & 95.46 & 93.90 & 94.90 & 95.52 & 95.60 & 94.08 & 95.26 & 95.10 & 95.52 & 93.20 & 94.78 \\
\hline & & & 1 & 95.54 & 95.54 & 94.18 & 94.92 & 95.34 & 95.52 & 93.88 & 94.98 & 95.04 & 95.06 & 93.20 & 94.42 \\
\hline & & & 3 & 95.68 & 95.68 & 94.32 & 95.02 & 95.28 & 95.46 & 94.14 & 94.78 & 95.68 & 95.62 & 94.06 & 94.88 \\
\hline & & 5 & 0.5 & 95.22 & 95.42 & 93.72 & 94.64 & 95.92 & 96.04 & 94.34 & 95.58 & 95.70 & 95.68 & 93.76 & 95.18 \\
\hline & & & 1 & 95.66 & 95.62 & 94.38 & 95.18 & 95.46 & 95.52 & 93.84 & 94.90 & 95.30 & 95.36 & 93.60 & 94.96 \\
\hline & & & 3 & 95.86 & 96.02 & 94.56 & 95.26 & 95.62 & 95.60 & 93.92 & 94.92 & 96.40 & 96.30 & 94.44 & 95.58 \\
\hline
\end{tabular}


Table 2. Empirical coverage probabilities of four $95 \%$ simultaneous confidence regions for $\phi$ and $\psi$ under various settings with $n=150$.

\begin{tabular}{|c|c|c|c|c|c|c|c|c|c|c|c|c|c|c|c|}
\hline \multirow[b]{2}{*}{$r$} & \multirow[b]{2}{*}{$\lambda$} & \multirow[b]{2}{*}{$\mu_{R}$} & \multirow[b]{2}{*}{$\sigma_{R}^{2}$} & \multicolumn{4}{|c|}{$n_{E}: n_{R}: n_{P}=1: 1: 1$} & \multicolumn{4}{|c|}{$n_{E}: n_{R}: n_{P}=2: 2: 1$} & \multicolumn{4}{|c|}{$n_{E}: n_{R}: n_{P}=3: 2: 1$} \\
\hline & & & & GF & HG & WB & PB & GF & HG & WB & PB & GF & HG & WB & PB \\
\hline \multirow[t]{18}{*}{0.3} & \multirow[t]{9}{*}{0.6} & \multirow[t]{3}{*}{3} & 0.5 & 95.26 & 95.24 & 94.88 & 95.22 & 95.56 & 95.60 & 95.16 & 95.54 & 94.72 & 94.88 & 93.90 & 94.66 \\
\hline & & & 1 & 94.66 & 94.70 & 94.10 & 94.46 & 94.68 & 94.86 & 94.06 & 94.44 & 95.24 & 95.32 & 94.42 & 94.88 \\
\hline & & & 3 & 95.44 & 95.50 & 95.02 & 95.30 & 95.46 & 95.64 & 95.04 & 95.30 & 95.74 & 95.74 & 94.96 & 95.54 \\
\hline & & \multirow[t]{3}{*}{4} & 0.5 & 95.02 & 95.08 & 94.50 & 94.76 & 94.78 & 94.92 & 94.26 & 94.70 & 95.42 & 95.38 & 94.72 & 95.38 \\
\hline & & & 1 & 96.02 & 96.16 & 95.56 & 95.76 & 95.44 & 95.42 & 94.72 & 95.18 & 95.90 & 95.88 & 95.12 & 95.56 \\
\hline & & & 3 & 95.42 & 95.42 & 94.74 & 95.10 & 95.02 & 95.06 & 94.48 & 94.88 & 95.20 & 95.34 & 94.66 & 94.98 \\
\hline & & \multirow[t]{3}{*}{5} & 0.5 & 95.20 & 95.22 & 94.80 & 95.10 & 94.96 & 95.12 & 94.60 & 94.98 & 95.24 & 95.44 & 94.66 & 95.06 \\
\hline & & & 1 & 94.66 & 94.76 & 94.26 & 94.54 & 95.08 & 95.20 & 94.52 & 94.90 & 95.50 & 95.48 & 94.86 & 95.30 \\
\hline & & & 3 & 94.82 & 94.82 & 94.12 & 94.42 & 95.50 & 95.56 & 94.84 & 95.24 & 95.80 & 95.66 & 95.20 & 95.58 \\
\hline & \multirow[t]{9}{*}{0.7} & \multirow[t]{3}{*}{3} & 0.5 & 95.16 & 95.28 & 94.74 & 95.00 & 95.70 & 95.66 & 95.02 & 95.64 & 95.04 & 95.14 & 94.50 & 94.96 \\
\hline & & & 1 & 94.92 & 94.96 & 94.18 & 94.70 & 94.92 & 94.94 & 94.38 & 94.62 & 94.76 & 94.86 & 93.98 & 94.46 \\
\hline & & & 3 & 95.48 & 95.42 & 94.94 & 95.20 & 95.08 & 95.14 & 94.60 & 94.88 & 95.40 & 95.52 & 94.98 & 95.26 \\
\hline & & \multirow[t]{3}{*}{4} & 0.5 & 94.82 & 94.88 & 94.20 & 94.68 & 94.96 & 94.92 & 94.34 & 94.80 & 94.60 & 94.78 & 93.94 & 94.28 \\
\hline & & & 1 & 94.50 & 94.56 & 94.08 & 94.30 & 95.46 & 95.50 & 94.78 & 95.08 & 95.10 & 95.22 & 94.32 & 94.88 \\
\hline & & & 3 & 94.98 & 95.06 & 94.50 & 94.70 & 95.32 & 95.30 & 94.66 & 95.22 & 95.76 & 95.68 & 95.06 & 95.38 \\
\hline & & \multirow[t]{3}{*}{5} & 0.5 & 95.14 & 95.20 & 94.64 & 95.06 & 95.36 & 95.32 & 94.78 & 95.08 & 95.22 & 95.26 & 94.54 & 95.08 \\
\hline & & & 1 & 95.36 & 95.56 & 95.00 & 95.26 & 95.04 & 95.18 & 94.64 & 94.96 & 95.38 & 95.32 & 94.56 & 95.12 \\
\hline & & & 3 & 95.16 & 95.06 & 94.56 & 94.88 & 95.24 & 95.26 & 94.62 & 94.86 & 95.42 & 95.52 & 94.86 & 95.12 \\
\hline \multirow[t]{18}{*}{0.6} & \multirow[t]{9}{*}{0.3} & 3 & 0.5 & 95.46 & 95.52 & 94.86 & 95.28 & 95.24 & 95.24 & 94.46 & 95.00 & 94.82 & 94.88 & 94.16 & 94.82 \\
\hline & & & 1 & 95.06 & 95.20 & 94.58 & 94.94 & 95.08 & 95.08 & 94.56 & 94.98 & 95.16 & 95.04 & 94.42 & 94.86 \\
\hline & & & 3 & 95.14 & 95.04 & 94.70 & 94.92 & 94.84 & 94.78 & 94.22 & 94.54 & 95.46 & 95.54 & 94.78 & 95.26 \\
\hline & & 4 & 0.5 & 95.08 & 95.20 & 94.80 & 95.14 & 95.24 & 95.38 & 94.74 & 95.14 & 95.04 & 95.02 & 94.36 & 94.92 \\
\hline & & & 1 & 95.40 & 95.42 & 94.92 & 95.10 & 95.50 & 95.62 & 94.92 & 95.28 & 95.30 & 95.24 & 94.40 & 95.08 \\
\hline & & & 3 & 95.28 & 95.34 & 94.78 & 95.22 & 95.00 & 95.18 & 94.38 & 94.82 & 95.18 & 95.28 & 94.62 & 95.06 \\
\hline & & 5 & 0.5 & 95.52 & 95.44 & 94.98 & 95.34 & 94.72 & 94.66 & 93.98 & 94.42 & 94.84 & 94.86 & 94.22 & 94.58 \\
\hline & & & 1 & 95.20 & 95.26 & 94.66 & 95.00 & 95.54 & 95.48 & 94.76 & 95.08 & 95.30 & 95.20 & 94.66 & 95.04 \\
\hline & & & 3 & 95.32 & 95.32 & 94.82 & 95.08 & 95.60 & 95.66 & 94.94 & 95.32 & 95.58 & 95.60 & 95.02 & 95.36 \\
\hline & 0.4 & 3 & 0.5 & 95.46 & 95.52 & 94.94 & 95.16 & 94.76 & 94.80 & 94.08 & 94.68 & 94.48 & 94.48 & 93.68 & 94.30 \\
\hline & & & 1 & 94.82 & 94.84 & 94.32 & 94.56 & 94.68 & 94.80 & 93.82 & 94.54 & 95.64 & 95.64 & 94.88 & 95.38 \\
\hline & & & 3 & 95.16 & 95.28 & 94.46 & 94.92 & 94.86 & 94.94 & 94.32 & 94.66 & 95.26 & 95.32 & 94.68 & 94.94 \\
\hline & & 4 & 0.5 & 95.54 & 95.70 & 94.92 & 95.32 & 94.82 & 94.82 & 94.22 & 94.56 & 94.68 & 94.64 & 94.10 & 94.50 \\
\hline & & & 1 & 95.04 & 95.12 & 94.48 & 94.88 & 95.04 & 95.06 & 94.52 & 94.94 & 94.84 & 94.82 & 93.90 & 94.40 \\
\hline & & & 3 & 95.06 & 95.06 & 94.62 & 94.88 & 95.40 & 95.36 & 94.86 & 95.04 & 95.38 & 95.46 & 94.82 & 95.14 \\
\hline & & 5 & 0.5 & 95.68 & 95.62 & 95.22 & 95.62 & 94.90 & 94.98 & 94.20 & 94.64 & 95.74 & 95.72 & 95.12 & 95.58 \\
\hline & & & 1 & 95.22 & 95.32 & 94.90 & 95.10 & 95.16 & 95.20 & 94.46 & 94.86 & 94.52 & 94.54 & 93.96 & 94.40 \\
\hline & & & 3 & 94.88 & 95.08 & 94.50 & 94.78 & 95.54 & 95.64 & 95.08 & 95.36 & 94.92 & 94.94 & 94.14 & 94.50 \\
\hline 1.0 & 0.0 & 3 & 0.5 & 95.36 & 95.54 & 94.80 & 95.22 & 95.12 & 95.10 & 94.42 & 94.92 & 95.06 & 95.14 & 94.44 & 95.00 \\
\hline & & & 1 & 94.60 & 94.78 & 93.94 & 94.46 & 95.20 & 95.40 & 94.80 & 95.12 & 95.14 & 95.24 & 94.34 & 94.86 \\
\hline & & & 3 & 95.48 & 95.58 & 95.08 & 95.32 & 95.66 & 95.64 & 95.10 & 95.40 & 95.60 & 95.44 & 94.74 & 95.28 \\
\hline & & 4 & 0.5 & 95.36 & 95.32 & 94.78 & 95.06 & 95.00 & 95.02 & 94.40 & 94.80 & 95.04 & 95.16 & 94.44 & 94.86 \\
\hline & & & 1 & 95.18 & 95.14 & 94.66 & 94.90 & 95.08 & 95.14 & 94.48 & 94.96 & 95.22 & 95.34 & 94.66 & 95.18 \\
\hline & & & 3 & 95.64 & 95.60 & 95.26 & 95.50 & 95.06 & 95.10 & 94.78 & 94.92 & 95.38 & 95.44 & 94.88 & 95.18 \\
\hline & & 5 & 0.5 & 95.00 & 95.00 & 94.50 & 94.80 & 95.26 & 95.30 & 94.54 & 95.22 & 95.34 & 95.44 & 94.72 & 95.22 \\
\hline & & & 1 & 95.54 & 95.66 & 95.18 & 95.44 & 95.40 & 95.54 & 94.66 & 95.04 & 95.34 & 95.34 & 94.68 & 95.10 \\
\hline & & & 3 & 95.04 & 95.12 & 94.44 & 94.70 & 95.12 & 95.20 & 94.62 & 94.88 & 95.44 & 95.48 & 94.80 & 95.16 \\
\hline
\end{tabular}


Table 3. Empirical coverage probabilities of four $95 \%$ simultaneous confidence regions for $\phi$ and $\psi$ under various settings with $n=300$.

\begin{tabular}{|c|c|c|c|c|c|c|c|c|c|c|c|c|c|c|c|}
\hline \multirow[b]{2}{*}{$r$} & \multirow[b]{2}{*}{$\lambda$} & \multirow[b]{2}{*}{$\mu_{R}$} & \multirow[b]{2}{*}{$\sigma_{R}^{2}$} & \multicolumn{4}{|c|}{$n_{E}: n_{R}: n_{P}=1: 1: 1$} & \multicolumn{4}{|c|}{$n_{E}: n_{R}: n_{P}=2: 2: 1$} & \multicolumn{4}{|c|}{$n_{E}: n_{R}: n_{P}=3: 2: 1$} \\
\hline & & & & GF & HG & WB & PB & GF & HG & WB & PB & GF & HG & WB & PB \\
\hline \multirow[t]{18}{*}{0.3} & 0.6 & 3 & 0.5 & 95.08 & 95.02 & 94.92 & 94.96 & 95.02 & 95.02 & 94.66 & 95.02 & 95.22 & 95.34 & 94.98 & 95.20 \\
\hline & & & 1 & 95.58 & 95.64 & 95.40 & 95.46 & 94.84 & 94.82 & 94.54 & 94.76 & 95.16 & 95.20 & 94.94 & 95.12 \\
\hline & & & 3 & 94.48 & 94.50 & 94.14 & 94.24 & 95.26 & 95.30 & 95.04 & 95.20 & 95.56 & 95.58 & 95.22 & 95.38 \\
\hline & & 4 & 0.5 & 95.40 & 95.38 & 95.12 & 95.28 & 94.76 & 94.84 & 94.42 & 94.72 & 94.18 & 94.38 & 93.92 & 94.18 \\
\hline & & & 1 & 94.94 & 94.92 & 94.70 & 94.88 & 94.60 & 94.64 & 94.32 & 94.44 & 95.50 & 95.42 & 95.10 & 95.32 \\
\hline & & & 3 & 95.32 & 95.32 & 94.88 & 95.16 & 95.24 & 95.28 & 95.10 & 95.16 & 94.74 & 94.82 & 94.50 & 94.70 \\
\hline & & 5 & 0.5 & 95.08 & 95.14 & 94.88 & 95.02 & 94.94 & 94.92 & 94.60 & 94.86 & 95.08 & 95.16 & 94.76 & 94.98 \\
\hline & & & 1 & 95.18 & 95.18 & 94.84 & 95.00 & 95.42 & 95.32 & 95.14 & 95.36 & 95.72 & 95.72 & 95.40 & 95.60 \\
\hline & & & 3 & 94.74 & 94.82 & 94.48 & 94.78 & 95.28 & 95.28 & 94.96 & 95.12 & 94.92 & 94.84 & 94.62 & 94.80 \\
\hline & 0.7 & 3 & 0.5 & 95.04 & 95.08 & 94.86 & 94.92 & 94.84 & 94.84 & 94.54 & 94.76 & 95.28 & 95.26 & 95.04 & 95.16 \\
\hline & & & 1 & 95.58 & 95.62 & 95.30 & 95.44 & 95.10 & 94.98 & 94.86 & 94.94 & 95.46 & 95.44 & 95.12 & 95.36 \\
\hline & & & 3 & 95.32 & 95.34 & 94.98 & 95.14 & 95.50 & 95.48 & 95.28 & 95.40 & 95.48 & 95.46 & 95.12 & 95.30 \\
\hline & & 4 & 0.5 & 94.94 & 94.92 & 94.70 & 94.80 & 94.78 & 94.90 & 94.54 & 94.76 & 95.22 & 95.12 & 94.86 & 95.16 \\
\hline & & & 1 & 95.42 & 95.46 & 95.32 & 95.42 & 95.12 & 95.16 & 94.92 & 95.20 & 95.18 & 95.18 & 94.92 & 95.12 \\
\hline & & & 3 & 95.14 & 95.34 & 95.02 & 95.02 & 94.72 & 94.74 & 94.66 & 94.72 & 95.22 & 95.18 & 94.96 & 95.10 \\
\hline & & 5 & 0.5 & 94.86 & 94.92 & 94.76 & 94.92 & 94.98 & 95.00 & 94.78 & 94.90 & 95.20 & 95.26 & 94.80 & 95.14 \\
\hline & & & 1 & 95.10 & 95.14 & 94.88 & 95.10 & 95.22 & 95.16 & 94.98 & 95.12 & 95.02 & 95.04 & 94.52 & 94.80 \\
\hline & & & 3 & 95.40 & 95.32 & 95.08 & 95.38 & 95.26 & 95.40 & 94.94 & 95.08 & 94.94 & 94.94 & 94.62 & 94.72 \\
\hline \multirow[t]{18}{*}{0.6} & 0.3 & 3 & 0.5 & 95.56 & 95.70 & 95.36 & 95.66 & 94.92 & 95.06 & 94.68 & 94.94 & 94.46 & 94.60 & 94.08 & 94.34 \\
\hline & & & 1 & 95.30 & 95.26 & 95.06 & 95.22 & 95.68 & 95.76 & 95.34 & 95.58 & 95.06 & 95.24 & 94.90 & 95.12 \\
\hline & & & 3 & 94.88 & 95.00 & 94.76 & 94.86 & 95.14 & 95.20 & 94.90 & 95.06 & 94.88 & 94.94 & 94.64 & 94.92 \\
\hline & & 4 & 0.5 & 95.38 & 95.40 & 95.08 & 95.24 & 95.04 & 95.20 & 94.82 & 95.06 & 94.98 & 94.94 & 94.58 & 94.72 \\
\hline & & & 1 & 94.92 & 95.00 & 94.64 & 94.82 & 94.84 & 94.92 & 94.60 & 94.86 & 95.04 & 95.10 & 94.82 & 94.96 \\
\hline & & & 3 & 95.50 & 95.52 & 95.26 & 95.32 & 95.24 & 95.26 & 95.10 & 95.24 & 94.84 & 94.86 & 94.56 & 94.72 \\
\hline & & 5 & 0.5 & 95.50 & 95.56 & 95.34 & 95.36 & 94.94 & 94.86 & 94.72 & 94.84 & 95.02 & 95.16 & 94.80 & 94.96 \\
\hline & & & 1 & 95.10 & 94.92 & 94.92 & 94.92 & 95.38 & 95.40 & 95.14 & 95.26 & 94.72 & 94.82 & 94.38 & 94.66 \\
\hline & & & 3 & 95.00 & 95.06 & 94.84 & 94.88 & 94.92 & 94.96 & 94.68 & 94.88 & 94.94 & 95.00 & 94.52 & 94.66 \\
\hline & 0.4 & 3 & 0.5 & 95.18 & 95.26 & 94.86 & 95.02 & 95.10 & 95.20 & 94.86 & 95.04 & 94.92 & 94.94 & 94.48 & 94.72 \\
\hline & & & 1 & 95.16 & 95.18 & 94.94 & 95.10 & 94.92 & 94.98 & 94.70 & 94.80 & 94.72 & 94.76 & 94.42 & 94.64 \\
\hline & & & 3 & 94.52 & 94.66 & 94.30 & 94.24 & 94.88 & 94.98 & 94.74 & 94.72 & 95.36 & 95.34 & 95.06 & 95.14 \\
\hline & & 4 & 0.5 & 95.26 & 95.24 & 94.96 & 95.18 & 95.12 & 95.06 & 94.82 & 95.02 & 95.06 & 95.10 & 94.78 & 95.06 \\
\hline & & & 1 & 95.34 & 95.38 & 95.22 & 95.22 & 95.22 & 95.18 & 94.88 & 95.18 & 94.96 & 94.94 & 94.56 & 94.76 \\
\hline & & & 3 & 94.96 & 94.80 & 94.62 & 94.78 & 94.52 & 94.64 & 94.26 & 94.52 & 95.20 & 95.18 & 94.94 & 95.02 \\
\hline & & 5 & 0.5 & 94.46 & 94.58 & 94.38 & 94.48 & 95.42 & 95.38 & 94.94 & 95.30 & 95.02 & 95.08 & 94.66 & 94.94 \\
\hline & & & 1 & 95.26 & 95.38 & 95.06 & 95.26 & 94.74 & 94.92 & 94.30 & 94.44 & 94.56 & 94.74 & 94.34 & 94.54 \\
\hline & & & 3 & 95.20 & 95.34 & 94.88 & 95.08 & 95.12 & 95.14 & 94.82 & 94.90 & 94.70 & 94.80 & 94.32 & 94.56 \\
\hline \multirow[t]{9}{*}{1.0} & 0.0 & 3 & 0.5 & 95.26 & 95.42 & 94.98 & 95.20 & 95.14 & 95.24 & 94.76 & 94.96 & 95.42 & 95.50 & 95.18 & 95.36 \\
\hline & & & 1 & 95.48 & 95.46 & 95.28 & 95.36 & 94.30 & 94.30 & 93.90 & 94.16 & 94.96 & 95.00 & 94.56 & 94.80 \\
\hline & & & 3 & 94.92 & 94.98 & 94.88 & 95.06 & 94.96 & 94.96 & 94.70 & 94.94 & 94.68 & 94.72 & 94.36 & 94.54 \\
\hline & & 4 & 0.5 & 94.90 & 94.86 & 94.56 & 94.72 & 95.30 & 95.34 & 95.06 & 95.16 & 95.32 & 95.48 & 95.00 & 95.22 \\
\hline & & & 1 & 95.38 & 95.48 & 95.30 & 95.38 & 94.98 & 95.02 & 94.82 & 95.02 & 94.82 & 95.04 & 94.58 & 94.90 \\
\hline & & & 3 & 95.04 & 95.06 & 94.82 & 95.02 & 94.88 & 94.98 & 94.68 & 94.76 & 95.12 & 95.20 & 94.64 & 94.82 \\
\hline & & 5 & 0.5 & 95.24 & 95.32 & 95.00 & 95.14 & 95.06 & 95.06 & 94.80 & 95.02 & 95.16 & 95.20 & 94.94 & 95.18 \\
\hline & & & 1 & 94.50 & 94.50 & 94.26 & 94.36 & 95.10 & 95.10 & 94.78 & 95.04 & 94.60 & 94.68 & 94.42 & 94.48 \\
\hline & & & 3 & 94.82 & 94.78 & 94.48 & 94.66 & 94.64 & 94.60 & 94.40 & 94.56 & 94.58 & 94.60 & 94.08 & 94.38 \\
\hline
\end{tabular}


Table 4. The required sample sizes $n$ for true power $1-\beta=95 \%$ and its empirical powers $p^{*}$ under various settings with $\epsilon=0.4$.

\begin{tabular}{|c|c|c|c|c|c|c|c|c|c|c|c|c|c|c|c|c|c|c|c|}
\hline \multirow[b]{3}{*}{$r$} & \multirow[b]{3}{*}{$\lambda$} & \multirow[b]{3}{*}{$\mu_{R}$} & \multirow[b]{3}{*}{$\sigma_{R}^{2}$} & \multicolumn{8}{|c|}{$n_{E}: n_{R}: n_{P}=1: 1: 1$} & \multicolumn{8}{|c|}{$n_{E}: n_{R}: n_{P}=2: 1: 1$} \\
\hline & & & & \multicolumn{2}{|c|}{ GF-SCI } & \multicolumn{2}{|c|}{ HG-SCI } & \multicolumn{2}{|c|}{ WB-SCI } & \multicolumn{2}{|c|}{ PB-SCI } & \multicolumn{2}{|c|}{ GF-SCI } & \multicolumn{2}{|c|}{ HG-SCI } & \multicolumn{2}{|c|}{ WB-SCI } & \multicolumn{2}{|c|}{ PB-SCI } \\
\hline & & & & $n_{0}$ & $p^{*}$ & $n_{0}$ & $p^{*}$ & $n_{0}$ & $p^{*}$ & $n_{0}$ & $p^{*}$ & $n_{0}$ & $p^{*}$ & $n_{0}$ & $p^{*}$ & $n_{0}$ & $p^{*}$ & $n_{0}$ & $p^{*}$ \\
\hline \multirow[t]{18}{*}{0.3} & 0.6 & 3 & 0.5 & 486 & 95.78 & 486 & 95.70 & 450 & 94.92 & 468 & 95.00 & 548 & 95.20 & 548 & 95.08 & 548 & 95.20 & 548 & 95.22 \\
\hline & & & 1 & 525 & 4.74 & 525 & 4.86 & 525 & 5.06 & 525 & 4.98 & 648 & 95.64 & 648 & 5.60 & 600 & 4.04 & 600 & \\
\hline & & & 3 & 786 & 94.84 & 786 & 94.70 & 750 & 94.32 & 786 & 95.38 & 1000 & 95.64 & 1000 & 95.56 & 948 & 4.88 & 1000 & 5.70 \\
\hline & & 4 & 0.5 & 486 & 95.52 & 486 & 95.48 & 450 & 94.12 & 450 & 94.40 & 548 & 95.26 & 548 & 95.28 & 500 & 94.18 & 548 & 95.54 \\
\hline & & & 1 & 525 & 94.60 & 525 & 94.68 & 525 & 95.10 & 525 & 95.22 & 600 & 94.34 & 600 & 94.32 & 600 & 94.58 & 600 & 94.34 \\
\hline & & & 3 & 750 & & 750 & & 750 & & 786 & & 1000 & & 1000 & & 1000 & & 948 & 4.64 \\
\hline & & 5 & 0.5 & 450 & 94.20 & 450 & & 450 & & 450 & & 500 & 94.10 & 548 & 95.52 & 500 & 4.40 & 548 & \\
\hline & & & 1 & 525 & 94.72 & 525 & 94.76 & 525 & 94.94 & 525 & 94.76 & 600 & 94.42 & 596 & 94.06 & 600 & 4.42 & 600 & \\
\hline & & & 3 & 825 & 95.96 & 786 & 94.88 & 825 & 95.92 & 825 & 95.86 & 1000 & 95.90 & 948 & 94.80 & 948 & 5.12 & 1000 & \\
\hline & 0.7 & 3 & 0.5 & 450 & 94.12 & 468 & 94.90 & 450 & 94.30 & 450 & 94.04 & 548 & 95.18 & 548 & 95.28 & 524 & 94.04 & 500 & 94.14 \\
\hline & & & 1 & 525 & 94.40 & 525 & 94.50 & 525 & & 525 & & 600 & 94.10 & 600 & 94.48 & 600 & & 600 & 94.66 \\
\hline & & & 3 & 786 & & 825 & & 25 & & 786 & & 948 & & 1000 & & 1000 & & 948 & \\
\hline & & 4 & 0.5 & 486 & 95. & 486 & & 450 & & 450 & & 548 & & 548 & 48 & & 18 & 548 & \\
\hline & & & 1 & 525 & 94.92 & 525 & 94.86 & 525 & 94.58 & 525 & 94.04 & 648 & 95.46 & 648 & 95.50 & 600 & 5.00 & 600 & 4.08 \\
\hline & & & 3 & 825 & 95.86 & 825 & & 750 & 94.24 & 825 & & 1000 & 95.62 & 1000 & 95.66 & 1000 & & 1000 & \\
\hline & & 5 & 0.5 & 450 & 94.46 & 450 & 94.32 & 450 & 94.68 & 450 & 94 & 500 & 94.38 & 548 & 95.66 & 500 & 94.40 & 548 & 95.58 \\
\hline & & & 1 & 525 & 94.22 & 561 & & 525 & & 525 & & 600 & & 600 & & 600 & & 600 & 94.40 \\
\hline & & & 3 & 825 & & 750 & & & & & & 1000 & & 1000 & & & & 1000 & \\
\hline 0.6 & 0.3 & 3 & 0.5 & 45 & 94. & 450 & 94. & 450 & 94 & 450 & & 548 & 94.88 & 548 & 94. & & 78 & 548 & \\
\hline & & & 1 & 525 & & 525 & & 525 & & 525 & & 600 & 94.34 & 600 & 94.40 & & & 648 & \\
\hline & & & 3 & 825 & 95. & 825 & & 825 & & 786 & & 1000 & 95.62 & 1000 & 95.62 & 1000 & 95.92 & 1000 & 95.58 \\
\hline & & 4 & 0.5 & 486 & 95.76 & 486 & 95.72 & 486 & 95. & 450 & 94.26 & 500 & 94.36 & 500 & 94.26 & 500 & 94.18 & 548 & 95.20 \\
\hline & & & 1 & 525 & 94.72 & 525 & 94.60 & 525 & & 525 & & 600 & & 600 & & 00 & 94.34 & 600 & 94.14 \\
\hline & & & 3 & 786 & 94. & 786 & & 82 & & & & 1000 & & 1000 & & & & 948 & \\
\hline & & 5 & 0.5 & 450 & 94. & 486 & 95. & 450 & & 486 & & 548 & 95.52 & 548 & 95.62 & & 94.76 & 524 & \\
\hline & & & 1 & 525 & & 525 & 94. & 525 & & 525 & & 648 & 95.6 & 648 & 50 & & & 648 & \\
\hline & & & 3 & 825 & 95. & 825 & & 750 & 94 & 750 & & 948 & 95.12 & 1000 & 95.88 & 948 & 94.94 & 1000 & 95.56 \\
\hline & 0.4 & 3 & 0.5 & 486 & 95.24 & 486 & 95.18 & 450 & 94.48 & 450 & 94.46 & 500 & 94.12 & 548 & 95.34 & 500 & 94.26 & 548 & 94.98 \\
\hline & & & 1 & 525 & & 525 & & 525 & & 525 & & 600 & & 648 & & 600 & 94.36 & 648 & 95.86 \\
\hline & & & 3 & 825 & & 825 & & 825 & & 786 & & 1000 & & 1000 & & & & 1000 & \\
\hline & & 4 & 0.5 & 450 & 94.7 & 450 & 94.66 & 450 & 94.44 & 450 & & 548 & 95.24 & 548 & 95.20 & 548 & 95.50 & 548 & 95.52 \\
\hline & & & 1 & 525 & 94.5 & 525 & 94. & 525 & & 525 & 94.90 & 600 & 94.44 & 600 & 94.34 & 648 & 95.66 & 600 & 94.16 \\
\hline & & & 3 & 786 & 94.90 & 786 & 94.7 & 750 & 94. & 750 & & 1000 & 95.60 & 1000 & 95.60 & 1000 & 95.80 & 1000 & 95.90 \\
\hline & & 5 & 0.5 & 486 & 95.18 & 486 & 95.08 & 450 & 94.44 & 450 & 94.14 & 548 & 95.48 & 548 & 95.46 & 548 & 95.62 & 548 & 95.32 \\
\hline & & & 1 & 525 & 94.78 & 525 & & 525 & & 525 & & 600 & 94.36 & 600 & & 600 & 94.32 & 600 & 94.42 \\
\hline & & & 3 & 786 & 95.5 & 786 & & 786 & & 825 & & 1000 & 95.74 & 1000 & 95.86 & 1000 & 95.94 & 900 & 94.14 \\
\hline 1.0 & 0.0 & 3 & 0.5 & 450 & 94.2 & 450 & 94.08 & 450 & 94.40 & 468 & 94.8 & 548 & 95.06 & 548 & 95.02 & 548 & 95.50 & 500 & 94. \\
\hline & & & 1 & 525 & 94.5 & 525 & & 525 & & 525 & 94.64 & 600 & 94.20 & 600 & 94.20 & 60 & 94.72 & 600 & 94.44 \\
\hline & & & 3 & 825 & 95.7 & 825 & & 786 & 95. & 786 & & 1000 & 95.94 & 948 & 94.66 & 900 & 94.42 & 1000 & 95.54 \\
\hline & & 4 & 0.5 & 450 & 94.70 & 450 & 94.66 & 468 & 94.82 & 450 & 94.04 & 548 & 94.92 & 548 & 95.18 & 500 & 94.02 & 548 & 95.22 \\
\hline & & & 1 & 525 & 94.32 & 525 & 94.20 & 525 & 94.92 & 525 & 94.38 & 600 & 94.56 & 648 & 95.74 & 600 & 94.48 & 600 & 94.36 \\
\hline & & & 3 & 825 & 95.90 & 786 & 94.88 & 750 & 94.34 & 825 & 95.36 & 1000 & 95.80 & 1000 & 95.96 & 1000 & 95.46 & 1000 & 95.82 \\
\hline & & 5 & 0.5 & 486 & 95.14 & 486 & 95.08 & 450 & 94.46 & 486 & 95.84 & 548 & 95.30 & 548 & 95.20 & 500 & 94.10 & 548 & 95.24 \\
\hline & & & 1 & 525 & & 525 & & 525 & & 525 & & 648 & & & & & & 600 & \\
\hline & & & 3 & 825 & 95.74 & 825 & 95.64 & 750 & 94.54 & 825 & 95.74 & 1000 & 95.86 & 1000 & 95.88 & 1000 & 95.94 & 1000 & 95.88 \\
\hline
\end{tabular}


Table 4. Cont.

\begin{tabular}{|c|c|c|c|c|c|c|c|c|c|c|c|c|c|c|c|c|c|c|c|}
\hline \multirow[b]{3}{*}{$r$} & \multirow[b]{3}{*}{$\lambda$} & \multirow[b]{3}{*}{$\mu_{R}$} & \multirow[b]{3}{*}{$\sigma_{R}^{2}$} & \multicolumn{8}{|c|}{$n_{E}: n_{R}: n_{P}=1: 1: 1$} & \multicolumn{8}{|c|}{$n_{E}: n_{R}: n_{P}=2: 1: 1$} \\
\hline & & & & \multicolumn{2}{|c|}{ GF-SCI } & \multicolumn{2}{|c|}{ HG-SCI } & \multicolumn{2}{|c|}{ WB-SCI } & \multicolumn{2}{|c|}{ PB-SCI } & \multicolumn{2}{|c|}{ GF-SCI } & \multicolumn{2}{|c|}{ HG-SCI } & \multicolumn{2}{|c|}{ WB-SCI } & \multicolumn{2}{|c|}{ PB-SCI } \\
\hline & & & & $n_{0}$ & $p^{*}$ & $n_{0}$ & $p^{*}$ & $n_{0}$ & $p^{*}$ & $n_{0}$ & $p^{*}$ & $n_{0}$ & $p^{*}$ & $n_{0}$ & $p^{*}$ & $n_{0}$ & $p^{*}$ & $n_{0}$ & $p^{*}$ \\
\hline \multirow[t]{18}{*}{0.3} & 0.6 & 3 & 0.5 & 625 & 95.42 & 625 & 95.44 & 625 & 95.68 & 625 & 95.62 & 672 & 95.78 & 672 & 95.74 & 672 & 95.20 & 672 & 95.46 \\
\hline & & & 1 & 685 & 95.48 & 685 & & 685 & 95.44 & 655 & 4.68 & 750 & 95.62 & 750 & 95.46 & 750 & 95.80 & 750 & \\
\hline & & & 3 & 875 & 95.12 & 875 & & 875 & 95.20 & 875 & 94.28 & 1050 & 95.24 & 1050 & 95.32 & & 94.34 & 1050 & \\
\hline & & 4 & 0.5 & 625 & 95.30 & 625 & 95.22 & 625 & 95.46 & 625 & 94.98 & 672 & 95.48 & 672 & 95.36 & 672 & 95.92 & 636 & 94.72 \\
\hline & & & 1 & 685 & 95.34 & 685 & 95.36 & 625 & 94.12 & 685 & 95.12 & 750 & 95.08 & 750 & 95.06 & 750 & 95.60 & 750 & 95.82 \\
\hline & & & 3 & 875 & 94.82 & 875 & 94.84 & 875 & 94.48 & 875 & 94.42 & 1050 & 95.92 & 1050 & 95.96 & 972 & 94.70 & 1050 & 95.74 \\
\hline & & 5 & 0.5 & 625 & 95.36 & 625 & 95.36 & 625 & 95.48 & 625 & 95.02 & 672 & 95.48 & 672 & 95.52 & 672 & 95.70 & 672 & 95.86 \\
\hline & & & 1 & 685 & 95.52 & 685 & & 685 & & 685 & & 750 & & 750 & & 750 & 95.42 & 750 & 5.42 \\
\hline & & & 3 & 875 & 94.76 & 875 & 94. & 875 & 94.88 & 875 & 74 & 972 & 94.60 & 1050 & 95.76 & 1050 & 5.88 & 1014 & 5.46 \\
\hline & 0.7 & 3 & 0.5 & 625 & 95.18 & 625 & 95.28 & 625 & 95.40 & 625 & 95.10 & 672 & 95.62 & 672 & 95.68 & 672 & 95.50 & 636 & 4.74 \\
\hline & & & 1 & 685 & 95.52 & 685 & 95.62 & 685 & 95.48 & 685 & 95.60 & 750 & 95.66 & 750 & 95.52 & 750 & 5.92 & 750 & 78 \\
\hline & & & 3 & 875 & 94.72 & 875 & 94.88 & 875 & 94.68 & 875 & 94.46 & 1050 & 95.26 & 1050 & 95.38 & 1050 & 95.80 & 1050 & 95.82 \\
\hline & & 4 & 0.5 & 625 & 95.46 & 625 & 95.54 & 625 & 95.18 & 625 & 95.94 & 672 & 95.42 & 672 & 95.38 & 600 & 94.02 & 672 & 95.22 \\
\hline & & & 1 & 685 & 94.92 & 685 & & & & & & 750 & & 750 & & & & 750 & .56 \\
\hline & & & 3 & 875 & 94.7 & 875 & & 875 & 94.58 & 875 & & 1050 & & 1050 & 58 & 050 & 95.94 & 1014 & 46 \\
\hline & & 5 & 0.5 & 625 & 95.00 & 625 & 95.24 & 625 & 95.26 & 625 & 95.96 & 672 & 95.12 & 672 & 95.38 & 600 & 94.24 & 672 & 46 \\
\hline & & & 1 & 625 & 94.10 & 685 & & 685 & 95.92 & 685 & & 750 & 95.26 & 750 & 95.20 & 750 & 95.72 & 750 & 95 \\
\hline & & & 3 & 875 & 94.14 & 875 & 94.54 & 875 & 94.78 & 875 & 95. & 1050 & 95.66 & 1050 & 95.96 & 972 & 94.42 & 972 & 94.20 \\
\hline 0.6 & 0.3 & 3 & 0.5 & 625 & 95.14 & 625 & 94.98 & 625 & 95.48 & 625 & 95. & 672 & 95.72 & 672 & 95.66 & 672 & 95.56 & 672 & 95.60 \\
\hline & & & 1 & 655 & & & & & & & & 750 & & 750 & & & 5.44 & 750 & \\
\hline & & & 3 & 875 & 94. & 875 & & & & 875 & & 1050 & & 1050 & 95. & 50 & 5.96 & 972 & \\
\hline & & 4 & 0.5 & 625 & 95.2 & 625 & 95. & 625 & 95.36 & 625 & & 672 & & 672 & 95. & & 95.42 & 672 & \\
\hline & & & 1 & 625 & 94.50 & 625 & & 685 & 95.64 & 685 & & 750 & 95.76 & 750 & 95.64 & 750 & 95.28 & 750 & 95.64 \\
\hline & & & 3 & 875 & 94.84 & 875 & 94.62 & 875 & 95.20 & 875 & 95.24 & 1050 & 95.30 & 1050 & 95.36 & 1014 & 95.36 & 1050 & 95.68 \\
\hline & & 5 & 0.5 & 625 & 95.32 & 625 & 95.62 & 625 & 95.74 & 625 & & 600 & 94.20 & 672 & 94.88 & 672 & 95.70 & 672 & 95.76 \\
\hline & & & 1 & 625 & & 685 & & & & & & & & 750 & & & & 750 & \\
\hline & & & 3 & 875 & & & & & & 875 & & 1050 & 95.98 & 972 & 94.40 & 972 & 94.20 & 972 & \\
\hline & 0.4 & 3 & 0.5 & 625 & 95.06 & 625 & & 625 & 95.68 & 625 & & 672 & 95.40 & 672 & 95.68 & 67 & 95.78 & 672 & \\
\hline & & & 1 & 685 & 95. & 685 & & 685 & & 685 & & 750 & & 750 & 95.70 & 750 & 95.82 & 750 & 95.28 \\
\hline & & & 3 & 875 & 95.24 & 875 & 94.98 & 875 & 95.38 & 875 & 95.28 & 972 & 94.06 & 1050 & 95.92 & 972 & 94.62 & 1050 & 95.86 \\
\hline & & 4 & 0.5 & 625 & 95.16 & 625 & 94.96 & 625 & 95.86 & 625 & 95 & 672 & 48 & 672 & 95.38 & 672 & 95.54 & 672 & 95.42 \\
\hline & & & 1 & 685 & & 685 & & & & & & 750 & & 750 & & & & 750 & \\
\hline & & & 3 & 875 & 95. & 875 & & 875 & & 875 & & 1050 & 95.92 & 1050 & 95. & 97 & 95.18 & 1050 & \\
\hline & & 5 & 0.5 & 625 & 95.10 & 625 & 95.04 & 625 & 95.12 & 625 & 95. & 672 & 95.80 & 672 & 95.74 & 636 & 94.68 & 672 & 95.54 \\
\hline & & & 1 & 685 & 95.7 & 685 & 95. & 685 & 95. & 685 & & 750 & & 750 & 95.48 & 750 & 95.62 & 750 & 95.76 \\
\hline & & & 3 & 875 & 94.76 & 875 & 94.68 & 875 & 94.12 & 875 & 94.74 & 1050 & 95.76 & 1050 & 95.60 & 1014 & 95.44 & 1050 & 95.94 \\
\hline 1.0 & 0.0 & 3 & 0.5 & 625 & 95.48 & 625 & 94.76 & 625 & 95.48 & 625 & 95.34 & 672 & 95.06 & 672 & 95.20 & 672 & 95.66 & 600 & 94.22 \\
\hline & & & 1 & 685 & 95.12 & 685 & 95. & 685 & 95. & 685 & 95. & 750 & 95.24 & 750 & 95.18 & 750 & 95.82 & 750 & 95.56 \\
\hline & & & 3 & 875 & 94. & 875 & 95. & 875 & 94.92 & 875 & 95.22 & 1014 & 94.82 & 1050 & 95.00 & 972 & 94.16 & 972 & 94.48 \\
\hline & & 4 & 0.5 & 625 & 95.64 & 625 & 95. & 625 & 95.98 & 625 & 95.26 & 672 & 95.40 & 672 & 95.22 & 672 & 95.88 & 672 & 95.54 \\
\hline & & & 1 & 685 & 95.8 & 685 & & 685 & & 685 & & 750 & & 750 & & 750 & 95.20 & 750 & 95.82 \\
\hline & & & 3 & 875 & 95.42 & 875 & 94. & 875 & 94. & 875 & 94.46 & 1014 & 95.20 & 1050 & 95.68 & 972 & 94.16 & 972 & 94.76 \\
\hline & & 5 & 0.5 & 625 & 95.18 & 625 & 95.16 & 625 & 95.52 & 625 & 95.80 & 672 & 95.56 & 672 & 95.52 & 600 & 94.08 & 672 & 95.84 \\
\hline & & & 1 & 685 & 95.54 & 685 & 95. & 685 & 95.96 & 685 & 95.36 & 750 & 95.44 & 750 & 95.38 & 750 & 95.56 & 750 & 95.24 \\
\hline & & & 3 & 875 & 95.04 & 875 & 95.02 & 875 & 95.10 & 875 & 94.54 & 1050 & 95.48 & 1050 & 95.44 & 1050 & 95.66 & 972 & 94.20 \\
\hline
\end{tabular}


Table 5. The required sample sizes $n$ for true power $1-\beta=95 \%$ and its empirical powers $p^{*}$ under various settings with $\epsilon=0.6$.

\begin{tabular}{|c|c|c|c|c|c|c|c|c|c|c|c|c|c|c|c|c|c|c|c|}
\hline \multirow[b]{3}{*}{$r$} & \multirow[b]{3}{*}{$\lambda$} & \multirow[b]{3}{*}{$\mu_{R}$} & \multirow[b]{3}{*}{$\sigma_{R}^{2}$} & \multicolumn{8}{|c|}{$n_{E}: n_{R}: n_{P}=1: 1: 1$} & \multicolumn{8}{|c|}{$n_{E}: n_{R}: n_{P}=2: 1: 1$} \\
\hline & & & & \multicolumn{2}{|c|}{ GF-SCI } & \multicolumn{2}{|c|}{ HG-SCI } & \multicolumn{2}{|c|}{ WB-SCI } & \multicolumn{2}{|c|}{ PB-SCI } & \multicolumn{2}{|c|}{ GF-SCI } & \multicolumn{2}{|c|}{ HG-SCI } & \multicolumn{2}{|c|}{ WB-SCI } & \multicolumn{2}{|c|}{ PB-SCI } \\
\hline & & & & $n_{0}$ & $p^{*}$ & $n_{0}$ & $p^{*}$ & $n_{0}$ & $p^{*}$ & $n_{0}$ & $p^{*}$ & $n_{0}$ & $p^{*}$ & $n_{0}$ & $p^{*}$ & $n_{0}$ & $p^{*}$ & $n_{0}$ & $p^{*}$ \\
\hline \multirow[t]{18}{*}{0.3} & 0.6 & 3 & 0.5 & 207 & 94.90 & 207 & 94.82 & 225 & 95.98 & 207 & 94.14 & 248 & 95.34 & 248 & 95.42 & 248 & 95.64 & 248 & 95.80 \\
\hline & & & 1 & 225 & 4.02 & 25 & 94.10 & & 94.04 & 222 & 4.26 & 300 & 5.84 & & & 300 & .54 & 300 & \\
\hline & & & 3 & 357 & 95.10 & 375 & 95.86 & 336 & 94.38 & 375 & 95.68 & 448 & 95.54 & 448 & & 448 & 5.74 & 448 & 5.54 \\
\hline & & 4 & 0.5 & 225 & 95.86 & 225 & 95.96 & 207 & 95.10 & 207 & & 248 & 95.00 & 248 & 94.90 & 248 & 95.78 & 248 & \\
\hline & & & 1 & 243 & 95.54 & 243 & 95.50 & 222 & 94.18 & 243 & & 276 & 94.68 & 276 & 94.62 & 276 & 95.36 & 300 & 95.92 \\
\hline & & & 3 & 357 & & 357 & & & & 357 & & 448 & & 448 & & & & 424 & \\
\hline & & 5 & 0.5 & 225 & 95.80 & & 94.48 & & & 216 & & & & & & & & 248 & \\
\hline & & & 1 & 243 & 94.64 & 243 & 95.66 & 231 & 94.44 & 243 & & 276 & & 276 & & & & 276 & \\
\hline & & & 3 & 357 & 94.96 & & 95.68 & & 94.04 & 357 & & 448 & & & & & & 448 & \\
\hline & 0.7 & 3 & 0.5 & 225 & 95.70 & 207 & 94.30 & 207 & 94.60 & 207 & & 248 & & 248 & & & & 236 & \\
\hline & & & 1 & 243 & 95.18 & 243 & 94.86 & 243 & 95.22 & 243 & & 300 & & 276 & & & & 276 & \\
\hline & & & 3 & & & & & & & & & & & & & & & 424 & \\
\hline & & 4 & 0.5 & 225 & 95.92 & & 95. & & & 20 & & & & & & & & 236 & \\
\hline & & & 1 & 243 & 95.28 & 243 & 95.16 & 225 & 94.68 & 225 & 94. & 276 & & & & & & 276 & \\
\hline & & & 3 & 375 & 95.98 & & & & 94.04 & 336 & & 448 & & & & & & 424 & \\
\hline & & 5 & 0.5 & 225 & 95.78 & 225 & 95.76 & 207 & 94.46 & 225 & 95. & 248 & & & & & & 248 & \\
\hline & & & 1 & 243 & 94.72 & 243 & 94.94 & 243 & 95.44 & 261 & 95.82 & 276 & & & & & & 276 & \\
\hline & & & 3 & & & & & & & & & & & & & & & 424 & \\
\hline 0.6 & 0.3 & 3 & 0.5 & 20 & 94.52 & 225 & 95. & & & & & & & & & & & 248 & \\
\hline & & & 1 & 22 & 94.14 & 261 & 95. & & & 24 & & & & & & & & 276 & \\
\hline & & & 3 & 357 & 95.08 & & 95.2 & & & & & & & & & & & 424 & \\
\hline & & 4 & 0.5 & 225 & 95.96 & 225 & 95.94 & 207 & 94.92 & 207 & 94.80 & 248 & & 248 & & & & 248 & 95.02 \\
\hline & & & 1 & 243 & 95.42 & 243 & 95.50 & 225 & 94.28 & 243 & & 276 & & & & & & 276 & 94.82 \\
\hline & & & 3 & 37 & & & & & & 357 & & & & & & & & & \\
\hline & & 5 & 0.5 & 20 & 94.24 & & 95 & 20 & & 20 & & & & & & & & 248 & \\
\hline & & & 1 & 24 & 95.14 & 243 & 95. & 22 & 94.2 & 24 & & 30 & & & & & & 276 & \\
\hline & & & 3 & 357 & 95.58 & & & & & & & & & & & & & 448 & \\
\hline & 0.4 & 3 & 0.5 & 207 & 94.44 & & 95.58 & 207 & 95.14 & 207 & & 248 & & & & & & 248 & 95. \\
\hline & & & 1 & 243 & 94.80 & 243 & 95.18 & 243 & 95.42 & 243 & & 276 & & & & 76 & & 276 & 94.48 \\
\hline & & & 3 & 375 & 95.90 & & & & & 33 & & & & & & & & 448 & \\
\hline & & 4 & 0.5 & 216 & 95.22 & & & 20 & & 20 & & & & & & & & & \\
\hline & & & 1 & 222 & 94.26 & 222 & 94.18 & 243 & 94.92 & 243 & & 2 & 94. & & & 27 & & 276 & 94. \\
\hline & & & 3 & 375 & 95.96 & & 94.3 & & 94.26 & & & & & & & & & 424 & \\
\hline & & 5 & 0.5 & 225 & 95.90 & 225 & 95.74 & 207 & 94.78 & 207 & 94.32 & 248 & 95.26 & 48 & & 248 & 5.52 & 248 & 95.38 \\
\hline & & & 1 & 243 & 95.36 & 243 & 95.36 & 222 & 94.02 & 243 & 95.16 & 276 & & 76 & & 276 & & 276 & 95.36 \\
\hline & & & 3 & 357 & & $35 \%$ & & 336 & 94.02 & 33 & & 448 & & 4 & & 42 & & 448 & 95.52 \\
\hline 1.0 & 0.0 & 3 & 0.5 & 225 & 95.96 & 225 & 95. & $20 \pi$ & 94.68 & 207 & & 24 & & 2 & & 24 & & & 95.2 \\
\hline & & & 1 & 243 & 95.20 & & & 24 & & 24 & & 27 & & & & & & & 94.66 \\
\hline & & & 3 & 357 & & & & & & 35 & & & & & & & & 424 & 94.86 \\
\hline & & 4 & 0.5 & 207 & 94.18 & & 95.84 & 207 & & 207 & & & & & & & & 248 & \\
\hline & & & 1 & 243 & 94.82 & 243 & 95.24 & 243 & 95.32 & 243 & 95.34 & 300 & 95.96 & 276 & 94.82 & 276 & 95.28 & 276 & 95.18 \\
\hline & & & 3 & 375 & 95.86 & 375 & 95.90 & 336 & 94.38 & 375 & 95.68 & 448 & 95.94 & 4 & 95.78 & 424 & 94.94 & 448 & 95.72 \\
\hline & & 5 & 0.5 & 225 & 95.52 & 225 & 95.48 & 207 & 94.52 & 207 & 94.92 & 24 & 95.28 & 24 & 95.32 & 22 & 94.20 & 248 & 95.06 \\
\hline & & & 1 & 243 & 95.04 & 243 & 94.86 & 243 & 94.86 & 243 & & 276 & & 27 & & 276 & & 300 & 95.86 \\
\hline & & & 3 & 336 & 94.04 & 357 & 95.28 & 336 & 94.34 & 375 & 95.94 & 448 & 95.78 & 448 & 95.64 & 424 & 95.08 & 448 & 95.70 \\
\hline
\end{tabular}


Table 5. Cont.

\begin{tabular}{|c|c|c|c|c|c|c|c|c|c|c|c|c|c|c|c|c|c|c|c|}
\hline \multirow[b]{3}{*}{$r$} & \multirow[b]{3}{*}{$\lambda$} & \multirow[b]{3}{*}{$\mu_{R}$} & \multirow[b]{3}{*}{$\sigma_{R}^{2}$} & \multicolumn{8}{|c|}{$n_{E}: n_{R}: n_{P}=1: 1: 1$} & \multicolumn{8}{|c|}{$n_{E}: n_{R}: n_{P}=2: 1: 1$} \\
\hline & & & & \multicolumn{2}{|c|}{ GF-SCI } & \multicolumn{2}{|c|}{ HG-SCI } & \multicolumn{2}{|c|}{ WB-SCI } & \multicolumn{2}{|c|}{ PB-SCI } & \multicolumn{2}{|c|}{ GF-SCI } & \multicolumn{2}{|c|}{ HG-SCI } & \multicolumn{2}{|c|}{ WB-SCI } & \multicolumn{2}{|c|}{ PB-SCI } \\
\hline & & & & $n_{0}$ & $p^{*}$ & $n_{0}$ & $p^{*}$ & $n_{0}$ & $p^{*}$ & $n_{0}$ & $p^{*}$ & $n_{0}$ & $p^{*}$ & $n_{0}$ & $p^{*}$ & $n_{0}$ & $p^{*}$ & $n_{0}$ & $p^{*}$ \\
\hline \multirow[t]{18}{*}{0.3} & 0.6 & 3 & 0.5 & 280 & 95.40 & 280 & 95.10 & 280 & 95.86 & 280 & 95.32 & 300 & 95.70 & 300 & 95.26 & 300 & 95.60 & 300 & 95.68 \\
\hline & & & 1 & 310 & 95.78 & 310 & & & 95.70 & 310 & 5.74 & 336 & 95.50 & 336 & & 336 & 95.78 & 336 & \\
\hline & & & 3 & 405 & 95.58 & 405 & 95.26 & 370 & 94.22 & 405 & 95.32 & 450 & 95.08 & 450 & 95.30 & 450 & 95.42 & 450 & 5.04 \\
\hline & & 4 & 0.5 & 280 & 95.22 & 280 & 95.18 & 280 & 95.68 & 280 & 95.32 & 300 & 95.54 & 300 & 95.42 & 300 & 95.72 & 300 & 5.70 \\
\hline & & & 1 & 310 & 94.70 & 310 & 94.60 & 310 & 95.44 & 310 & 95.86 & 336 & 95.84 & 336 & 95.70 & 336 & 95.98 & 336 & 95.16 \\
\hline & & & 3 & 405 & 95.36 & 405 & & 375 & 94.56 & 405 & & 450 & 94.54 & 450 & 94.60 & 450 & 95.26 & 450 & 94.80 \\
\hline & & 5 & 0.5 & 280 & 95.02 & 280 & & 280 & & 280 & 95.44 & 300 & & 300 & & 300 & 95.72 & 300 & \\
\hline & & & 1 & 310 & 95.76 & 310 & & 310 & 95.86 & 310 & & 336 & & 336 & & & 95.82 & 336 & \\
\hline & & & 3 & 405 & 95.60 & 405 & 95.28 & 385 & 94.60 & 405 & 95.52 & 450 & 94.78 & 450 & 94.74 & 450 & 94.86 & 450 & 4.94 \\
\hline & 0.7 & 3 & 0.5 & 280 & 95.16 & 280 & 95.12 & 280 & 95.80 & 280 & 95.34 & 300 & 95.40 & 300 & 95.96 & 300 & 95.76 & 300 & 95. \\
\hline & & & 1 & 310 & 95.70 & 310 & 95.18 & 310 & 95.48 & 310 & & 336 & 95.26 & 336 & & 336 & 95.66 & 336 & 95.36 \\
\hline & & & 3 & 405 & 95.76 & 405 & & 405 & & 375 & & 450 & & 450 & & 450 & 95.66 & 450 & 95.00 \\
\hline & & 4 & 0.5 & 280 & 95. & 280 & & 280 & & 280 & & 300 & & 300 & & & 95.42 & 300 & \\
\hline & & & 1 & 310 & 94.86 & 3 & 94. & 315 & & 310 & & 336 & & 336 & & & 94.34 & 336 & \\
\hline & & & 3 & 405 & 95.22 & 405 & 95.28 & 375 & 94.16 & 405 & 95.56 & 450 & 95.28 & 450 & 95.34 & 450 & 95.24 & 450 & 95.54 \\
\hline & & 5 & 0.5 & 280 & 95.02 & 280 & 95.24 & 280 & 95.60 & 280 & 95.14 & 300 & 95.48 & 300 & 95. & 300 & 95.48 & 300 & 95.32 \\
\hline & & & 1 & 310 & 95.74 & 310 & 95.42 & 310 & 95.90 & 310 & 95. & 336 & 95.74 & 336 & & 336 & 95.36 & 336 & 95.22 \\
\hline & & & 3 & 370 & & 5 & & & & 375 & & 450 & & 450 & & 450 & 94.84 & 450 & 94.76 \\
\hline 0.6 & 0.3 & 3 & 0.5 & 280 & 95. & $2 \varepsilon$ & & & & 280 & & 300 & & 300 & & & 5.58 & 300 & .08 \\
\hline & & & 1 & 310 & & & & & & 310 & & 336 & & 336 & & & 5.66 & 336 & \\
\hline & & & 3 & 405 & 95.44 & 4( & & 395 & 94. & 405 & 95.64 & 450 & 95 & 450 & 94. & & 95.56 & 450 & \\
\hline & & 4 & 0.5 & 280 & 94.62 & 280 & & 280 & 95.16 & 280 & & 300 & 95.18 & 300 & 95.12 & 300 & 95.94 & 300 & 95.34 \\
\hline & & & 1 & 310 & 95.54 & 310 & 95.42 & 280 & 94.06 & 310 & 95.42 & 336 & 95.62 & 336 & & 336 & 95.68 & 336 & 95.22 \\
\hline & & & 3 & 405 & 95.42 & 4( & & 405 & & 405 & & 450 & & 450 & & 450 & 95.84 & 450 & 94.60 \\
\hline & & 5 & 0.5 & 280 & 94. & & & 280 & & 280 & & 300 & & 300 & & & & 300 & \\
\hline & & & 1 & 310 & 95. & & 95. & 310 & 95. & 280 & & 336 & 95 & 336 & & & 95.26 & 336 & 95. \\
\hline & & & 3 & 405 & 94.96 & & 94. & 375 & 94. & 375 & & 450 & 94.84 & 450 & 95. & 450 & 95.20 & 450 & 95.44 \\
\hline & 0.4 & 3 & 0.5 & 280 & 95.74 & 280 & & 280 & & 280 & & 300 & & 300 & 95.26 & 300 & 95.92 & 300 & 95.26 \\
\hline & & & 1 & 310 & 95.50 & 310 & 95.14 & 310 & 95.62 & 310 & 95.38 & 336 & 95.74 & 336 & 95.38 & 336 & 95.66 & 336 & 95.40 \\
\hline & & & 3 & 370 & 94.08 & 4( & & 385 & & 375 & & 450 & & 450 & & 450 & 94.96 & 450 & 94.68 \\
\hline & & 4 & 0.5 & 280 & 95. & 28 & & 280 & & 280 & & 300 & & 300 & & & 95.36 & 300 & 95.38 \\
\hline & & & 1 & 310 & 95. & 3 & 95. & 310 & 95. & 295 & 94. & 336 & 95.10 & 336 & 94. & 336 & 95.52 & 336 & 95.52 \\
\hline & & & 3 & 405 & 94.96 & 405 & 95. & 375 & 94. & 375 & 94.10 & 450 & 94.74 & 450 & 95.40 & 450 & 95.20 & 450 & 95.62 \\
\hline & & 5 & 0.5 & 280 & 95.00 & $2 \varepsilon$ & 94. & 280 & 95. & 280 & & 300 & 95.44 & 300 & 95.30 & 300 & 95.62 & 300 & 95.22 \\
\hline & & & 1 & 310 & 95.84 & 310 & 95.74 & 310 & 95.78 & 310 & 95.40 & 336 & 95.38 & 336 & 95.28 & 336 & 95.38 & 336 & 95.90 \\
\hline & & & 3 & 405 & 95. & 405 & & 375 & & 370 & & 450 & 66 & 450 & 60 & 450 & 94.96 & 450 & 94.36 \\
\hline 1.0 & 0.0 & 3 & 0.5 & 280 & 95. & 280 & 95. & 280 & 95. & 280 & & 300 & & 300 & & 30 & 95.72 & 300 & 95.62 \\
\hline & & & 1 & 310 & 95.8 & 31 & 95. & 310 & 95. & 310 & & 336 & & 336 & 95. & 336 & 95.34 & 336 & 95.38 \\
\hline & & & 3 & 405 & 95.74 & 405 & 95. & 405 & 95.40 & 405 & 95.58 & 450 & 95.26 & 450 & 94.90 & 450 & 95.36 & 450 & 95.42 \\
\hline & & 4 & 0.5 & 280 & 94.82 & 280 & & 280 & & 280 & & 300 & & 300 & & 300 & 95.94 & 300 & 95.18 \\
\hline & & & 1 & 310 & 95.28 & 310 & 95.8 & 310 & 95.64 & 310 & 95.38 & 336 & 94.50 & 336 & 95.78 & 336 & 95.86 & 312 & 94.12 \\
\hline & & & 3 & 405 & 95.54 & 405 & 95.80 & 375 & 94.02 & 375 & 94.24 & 450 & 94.74 & 450 & 94.86 & 450 & 95.38 & 450 & 95.32 \\
\hline & & 5 & 0.5 & 280 & 95.26 & 280 & 95.82 & 280 & 94.96 & 280 & 95.40 & 300 & 95.00 & 300 & 95.46 & 300 & 95.76 & 300 & 95.30 \\
\hline & & & 1 & 310 & 95.84 & 310 & 95.30 & 310 & 95.72 & 315 & 95.92 & 336 & 95.08 & 336 & 95.58 & 300 & 94.02 & 336 & 95.28 \\
\hline & & & 3 & 405 & 95.58 & 405 & 95.60 & 370 & 94.48 & 405 & 95.46 & 450 & 94.84 & 450 & 94.84 & 456 & 95.10 & 450 & 95.06 \\
\hline
\end{tabular}

\subsection{Application to Mildly Asthmatic Study}

As an illustration of the preceding proposed simultaneous confidence region, we consider a data set on "Mildly Asthmatic Study" taken from [5]. In the mildly asthmatic study, the primary outcome variable is the forced vital capacity (FVC), and the data set 
consists of experimental $\left(n_{E}=35\right)$, reference $\left(n_{R}=19\right)$, and placebo $\left(n_{P}=20\right)$ groups. The means, standard deviations, minimum and maximum values for the FVC are $4.32,1.16$, 0.8 and 6.52 for E group, respectively; 4.86, 1.03, 2.94 and 6.90 for R group, respectively; and 3.14, 0.97, 1.41 and 4.99 for placebo group, respectively. Since [5] did not present the details on the historical trial, Ghosh et al. [24] independently simulated 5000 historical data sets from the normal distribution with the specified parameters and sample sizes in applying their proposed approach to the assessment of the NI of experimental to reference in a three-arm trial. Unlike [24], we here divide the considered data set into the historical and current data sets, its $30 \%$ is taken as the historical data randomly sampled from the considered data set without replacement, and the rest is regarded as the current data. Thus, the above developed approach to determine NI margin is applied to the historical data, and the preceding proposed four simultaneous confidence regions for $\phi$ and $\psi$ are applied to the current data for simultaneously assessing both NI and assay sensitivity with the following specification: $\lambda=0.0,0.25,0.5,0.6,0.75$ and 0.9 together with $r=1-\lambda$ and $M=5000$. The lower limits of the approximate $95 \%$ one-sided simultaneous confidence region of $(\phi, \psi)$ for the GF-SCI, HG-SCI, WB-SCI and PB-SCI methods are presented in Table 6 . Examination of Table 6 shows that (i) the assay sensitivity can be established at the significance level $\alpha=5 \%$ in that lower limits of four confidence regions are greater than $\Delta$ regardless of the values of $\lambda$; (ii) the NI cannot be established at the significance level 5\% for the GF-SCI, HG-SCI, WB-SCI and PB-SCI methods in that lower limits of their corresponding confidence intervals are less than $-\Delta_{\mathrm{NI}}$ regardless of the values of $\lambda$, which is consistent with those given in [5].

Table 6. Lower limits of the approximate $95 \%$ one-sided simultaneous confidence regions of $(\phi, \psi)$ for the GF-SCI, HG-SCI, WB-SCI and PB-SCI methods in the mildly asthmatic study.

\begin{tabular}{ccccccccccc}
\hline & & & \multicolumn{2}{c}{ GF-SCI } & \multicolumn{2}{c}{ HG-SCI } & \multicolumn{2}{c}{ WB-SCI } & \multicolumn{2}{c}{ PB-SCI } \\
\cline { 5 - 11 }$\lambda$ & $\Delta_{\text {NI }}$ & $\boldsymbol{\Delta}$ & $\boldsymbol{\phi}$ & $\boldsymbol{\psi}$ & $\boldsymbol{\phi}$ & $\boldsymbol{\psi}$ & $\boldsymbol{\phi}$ & $\boldsymbol{\psi}$ & $\boldsymbol{\phi}$ & $\boldsymbol{\psi}$ \\
\hline 0 & 0.5359 & 0.5359 & -0.7413 & 1.7744 & -0.9295 & 1.4548 & -1.0836 & 1.3523 & -0.7403 & 1.7756 \\
0.25 & 0.9563 & 0.7172 & -0.5438 & 1.3784 & -0.7607 & 1.1330 & -0.8785 & 0.9472 & -0.5466 & 1.3748 \\
0.5 & 0.9288 & 0.4644 & -0.4105 & 1.5093 & -0.6439 & 1.2028 & -0.7676 & 1.0585 & -0.4095 & 1.5106 \\
0.6 & 0.9608 & 0.3843 & -0.3641 & 1.1416 & -0.6101 & 0.8457 & -0.7305 & 0.7259 & -0.3677 & 1.1376 \\
0.75 & 0.4707 & 0.1177 & -0.8854 & 1.7782 & -1.0603 & 1.4066 & -1.2334 & 1.3435 & -0.8849 & 1.7789 \\
0.9 & 0.7461 & 0.0746 & -0.5859 & 1.6179 & -0.7757 & 1.2700 & -0.9613 & 1.1762 & -0.5889 & 1.6145 \\
\hline
\end{tabular}

\section{Discussion}

This paper studied the confidence interval construction problem for simultaneously assessing the non-inferiority and assay sensitivity in a three-arm trial with normally distributed endpoints, in which the non-inferiority margin is defined as a pre-specified fixed difference between treatment effects of experimental and reference treatments in the considered historical placebo-controlled trial. The generalized fiducial pivotal quantity method together with the historical-trial-based approach were utilized to determine the non-inferiority margin. However, there was no closed-form for the non-inferiority margin via the historical-trial-based approach. To overcome the difficulty, a Monte Carlo resampling method was adopted to determine the non-inferiority margin based on the percentile point of the drawn observations. Based on the constructed non-inferiority margin, we developed four simultaneous confidence intervals for parameters $(\phi, \psi)$ based on the Waldtype statistic together with the Bonferroni method, generalized fiducial pivotal quantity method, hybrid generalized fiducial method incorporating the square-and-add method and generalized fiducial method, and parametric bootstrap resampling method. The flexible algorithms for computing empirical coverage probabilities for the proposed four simultaneous confidence intervals were also given. Simulation studies were conducted to investigate the performance of the proposed four simultaneous confidence intervals under various settings including the moderate and large sample sizes, balanced and unbalanced designs, different hypotheses including the HT hypothesis. Empirical results demonstrated that the 
GF-SCI and HG-SCI methods perform better than the WB-SCI and PB-SCI methods, and the GF-SCI, PB-SCI and HG-SCI methods had the same performance when the sample size was large (i.e., $n=150$ and 300) in terms of their empirical coverage probabilities. Hence, we recommended the usage of the hybrid generalized fiducial simultaneous confidence interval because its computational burden was less than that for the PB-SCI method, and the usage of the Wald-type Bonferroni simultaneous confidence interval when the sample size was large.

This paper did not consider the simultaneous confidence interval construction problem for simultaneously assessing both non-inferiority and assay sensitivity in a three-arm trial with normally distributed endpoints in the presence of missing endpoints, which are commonly encountered in the modern drug studies. We did not consider the usage of the prior information in determining the non-inferiority margin from the placebo-controlled data, which may improve the efficiency of the estimation for the non-inferiority margin.

Author Contributions: Conceptualization, N.T.; methodology, N.T. and F.L.; software, F.L.; validation, N.T.; formal analysis, N.T. and F.L.; investigation, F.L.; resources, N.T. and F.L.; data curation, N.T.; writing — original draft preparation, F.L.; writing—review and editing, N.T.; visualization, F.L.; supervision, N.T.; project administration, N.T.; funding acquisition, N.T. All authors have read and agreed to the published version of the manuscript.

Funding: This research was funded by the grants from the Key Projects of the National Natural Science Foundation of China (Grant No.: 11731101).

Institutional Review Board Statement: Not applicable.

Informed Consent Statement: Not applicable.

Data Availability Statement: The datasets generated and analysed are available from the corresponding author on reasonable request.

Conflicts of Interest: The authors declare no conflict of interest.

\section{Abbreviations}

The following abbreviations are used in this manuscript:

CI Confidence interval

GFPQ Generalized fiducial pivotal quantity

WB-SCI Wald-type Bonferroni simultaneous confidence region

GF-SCI Simultaneous generalized fiducial confidence region

ECP empirical coverage probability

HG-SCI Hybrid generalized fiducial simultaneous confidence region

PB-SCI Parametric bootstrap simultaneous confidence region

\section{Appendix A. Proof of Theorem 1}

Proof. Denote $\mathbf{S}_{\mathbf{n}}=\left(\bar{X}_{E}, \bar{X}_{R}, \bar{X}_{P}, S_{E}^{2}, S_{R}^{2}, S_{P}^{2}\right), \mathbf{m}=\left(\mu_{E}, \mu_{R}, \mu_{P}, \sigma_{E}^{2}, \sigma_{R}^{2}, \sigma_{P}^{2}\right)$, and $\Omega=\operatorname{diag}\left(\sigma_{E} / \sqrt{r_{E}}, \sigma_{R} / \sqrt{r_{R}}, \sigma_{P} / \sqrt{r_{P}}, \sqrt{2} \sigma_{E}^{2} / \sqrt{r_{E}}, \sqrt{2} \sigma_{R}^{2} / \sqrt{r_{R}}, \sqrt{2} \sigma_{P}^{2} / \sqrt{r_{P}}\right)$. The central limit theorem implies that $\sqrt{n}\left(\mathbf{S}_{\mathbf{n}}-\mathbf{m}\right) \stackrel{\mathrm{D}}{\rightarrow} \mathbf{\Omega Z}$ where $\mathbf{Z}=\left(Z_{1}, \ldots, Z_{6}\right)$ are i.i.d. $N(0,1)$ variables. By Skorohod's theorem (see Billingsley 1995) we can find a sequence $\overline{\mathbf{S}}_{\mathbf{n}}$ independent of $\mathbf{S}^{*}$ such that $\overline{\mathbf{S}}_{\mathbf{n}}$ has the same distribution as $\mathbf{S}$ and $\sqrt{n}\left(\overline{\mathbf{S}}_{\mathbf{n}}-\mathbf{m}\right) \rightarrow \mathbf{\Omega Z}$ almost surely, where $\mathbf{S}$ denote observable random vector and $\mathbf{S}^{*}$ represent an independent copy of S. In what follows we can therefore assume without loss of generality that

$$
\sqrt{n}\left(\mathbf{S}_{\mathbf{n}}-\mathbf{m}\right) \rightarrow \mathbf{\Omega Z} \text { a.s. }
$$


It follows from the Sluctsky's theorem that as $n \rightarrow \infty$

$$
\begin{aligned}
& \max \left(\frac{\hat{\phi}-R_{\phi}}{\sqrt{\operatorname{vâr}(\hat{\phi})}}, \frac{\hat{\psi}-R_{\psi}}{\sqrt{\operatorname{vâr}(\hat{\psi})}}\right) \\
& \rightarrow \max \left(\frac{\frac{\sigma_{E}}{\sqrt{r_{E}}} Z_{E}-\frac{\sigma_{R}}{\sqrt{r_{R}}} Z_{R}}{\sqrt{\frac{\sigma_{E}^{2}}{r_{E}}+\frac{\sigma_{R}^{2}}{r_{R}}}}, \frac{\frac{\sigma_{R}}{\sqrt{r_{R}}} Z_{R}-\frac{\sigma_{P}}{\sqrt{r_{P}}} Z_{P}}{\sqrt{\frac{\sigma_{R}^{2}}{r_{R}}+\frac{\sigma_{P}^{2}}{r_{P}}}}\right) \text { a.s. }
\end{aligned}
$$

where $Z_{k}$ denotes the standard normal distribution, for $k=E, R, P$ and the a.s. comes from the a.s. convergence in (A1).

Recall the definition of the percentile $d_{1-\alpha}$ above. Since the limiting distribution in (A2) is continuous, we have by the definition of convergence in distribution

$$
d_{1-\alpha} \rightarrow q_{1-\alpha}
$$

where $q_{1-\alpha}$ is the $1-\alpha$ percentile point of the limiting distribution in (A2).

Finally, realize that (A1) implies

$$
\begin{aligned}
& \frac{\hat{\phi}-\phi}{\sqrt{\operatorname{vâr}(\hat{\phi})}} \rightarrow \frac{\frac{\sigma_{E}}{\sqrt{r_{E}}} Z_{E}-\frac{\sigma_{R}}{\sqrt{r_{R}}} Z_{R}}{\sqrt{\frac{\sigma_{E}^{2}}{r_{E}}+\frac{\sigma_{R}^{2}}{r_{R}}}} \text { a.s. } \\
& \frac{\hat{\psi}-\psi}{\sqrt{\operatorname{vâr}(\hat{\psi})}} \rightarrow \frac{\frac{\sigma_{R}}{\sqrt{r_{R}}} Z_{R}-\frac{\sigma_{P}}{\sqrt{r_{P}}} Z_{P}}{\sqrt{\frac{\sigma_{R}^{2}}{r_{R}}+\frac{\sigma_{P}^{2}}{r_{P}}}} \text { a.s. }
\end{aligned}
$$

This, together with (A3) and some algebra gives

$$
\begin{aligned}
& P\left(\phi \geq L_{\phi}^{F}, \psi \geq L_{\psi}^{F}\right) \\
& =P\left(\max \left(\frac{\hat{\phi}-\phi}{\sqrt{\operatorname{vâr}(\hat{\phi})}}, \frac{\hat{\psi}-\psi}{\sqrt{\operatorname{vâr}(\hat{\psi})}}\right) \leq d_{1-\alpha}\right) \\
& \rightarrow P\left(\max \left(\frac{\frac{\sigma_{E}}{\sqrt{r_{E}}} Z_{E}-\frac{\sigma_{R}}{\sqrt{r_{R}}} Z_{R}}{\sqrt{\frac{\sigma_{E}^{2}}{r_{E}}+\frac{\sigma_{R}^{2}}{r_{R}}}}, \frac{\frac{\sigma_{R}}{\sqrt{r_{R}}} Z_{R}-\frac{\sigma_{P}}{\sqrt{r_{P}}} Z_{P}}{\sqrt{\frac{\sigma_{R}^{2}}{r_{R}}+\frac{\sigma_{P}^{2}}{r_{P}}}}\right) \leq q_{1-\alpha}\right) \\
& =1-\alpha
\end{aligned}
$$

as $n \rightarrow \infty$.

\section{Appendix B. Proof of Theorem 2}

Proof. Note that

$$
P\left(\phi \geq L_{\phi}^{B}, \psi \geq L_{\psi}^{B}\right)=P\left(T \leq q_{1-\alpha}^{B}\right)
$$

where $T=\max \left(\frac{\hat{\phi}-\phi}{\sqrt{\operatorname{var}(\hat{\phi})}}, \frac{\hat{\psi}-\psi}{\sqrt{\operatorname{var}(\hat{\psi})}}\right)$ and $q_{1-\alpha}^{B}$ be the $1-\alpha$ percentile point of bootstrap sample observations of $T_{B}^{P I}$, where $T_{B}^{P I}$ is given by (24). To show the above simultaneous CI has correct coverage probability asymptotically, it is sufficient to show that $T$ has the same limiting distribution as $T_{B}^{P I}$ when $n \rightarrow \infty$. This can be justified, according to 
continuous mapping theorem if we can show that $t_{\phi}, t_{\psi}$ and $t_{\phi}^{B}, t_{\psi}^{B}$ have the same limiting joint distribution, respectively. Note that

$$
\begin{aligned}
& t_{\phi}=\frac{\hat{\phi}-\phi}{\sqrt{\operatorname{vâr}(\hat{\phi})}}=\frac{\sqrt{n} \hat{\phi}-\sqrt{n} \phi}{\sqrt{n} \sqrt{\operatorname{var}(\hat{\phi})}}, t_{\psi}=\frac{\hat{\psi}-\psi}{\sqrt{\operatorname{vâr}(\hat{\psi})}}=\frac{\sqrt{n} \hat{\psi}-\sqrt{n} \psi}{\sqrt{n} \sqrt{\operatorname{var}(\hat{\psi})}} \\
& t_{\phi}^{B}=\frac{\left(\bar{X}_{E}^{B}-\bar{X}_{R}^{B}\right)}{\sqrt{\operatorname{vâr}_{B}(\hat{\phi})}}=\frac{\sqrt{n}\left(\bar{X}_{E}^{B}-\bar{X}_{R}^{B}\right)}{\sqrt{n} \sqrt{\operatorname{vâr}_{B}(\hat{\phi})}}, t_{\psi}^{B}=\frac{\left(\bar{X}_{R}^{B}-\bar{X}_{P}^{B}\right)}{\sqrt{\operatorname{vâr}_{B}(\hat{\psi})}}=\frac{\sqrt{n}\left(\bar{X}_{R}^{B}-\bar{X}_{P}^{B}\right)}{\sqrt{n} \sqrt{\operatorname{vâr}_{B}(\hat{\psi})}}
\end{aligned}
$$

From condition $\frac{n_{k}}{n} \rightarrow r_{k}$, we can obtain that $\sqrt{n}\left(\hat{\mu}_{k}-\mu_{k}\right) \rightarrow \mathcal{N}\left(0, \sigma_{k}^{2} / r_{k}\right)$ and $\sqrt{n}\left(\hat{\sigma}_{k}^{2}-\right.$ $\left.\sigma_{k}^{2}\right) \rightarrow \mathcal{N}\left(0,2 \sigma_{k}^{4} / r_{k}\right)$, for $k=P, R, E$. Therefore, by Slutsky's theorem, it follows that

$$
T \rightarrow \max \left\{\frac{\frac{\sigma_{E}}{\sqrt{r_{E}}} Z_{E}-\frac{\sigma_{R}}{\sqrt{r_{R}}} Z_{R}}{\sqrt{\frac{\sigma_{E}^{2}}{r_{E}}+\frac{\sigma_{R}^{2}}{r_{R}}}}, \frac{\frac{\sigma_{R}}{\sqrt{r_{R}}} Z_{R}-\frac{\sigma_{P}}{\sqrt{r_{P}}} Z_{P}}{\sqrt{\frac{\sigma_{R}^{2}}{r_{R}}+\frac{\sigma_{P}^{2}}{r_{P}}}}\right\}
$$

where $Z_{k}$ denotes the standard normal distribution, for $k=E, R, P$. To show that $t_{\phi}^{B}, t_{\psi}^{B}$ also weakly converges to the same joint distribution, we first note that $\bar{X}_{k}^{B} \sim \frac{s_{k}}{\sqrt{n_{k}}} \mathcal{N}(0,1)$, $S_{k, B}^{2} \sim \frac{s_{k}^{2}}{n_{k}-1} \chi^{2}\left(n_{k}-1\right)$ for $k=P, R, E$ and they are independent. Therefore, by using the similar approach, we can obtain that

$$
T_{B}^{P I} \rightarrow \max \left\{\frac{\frac{\sigma_{E}}{\sqrt{r_{E}}} Z_{E}-\frac{\sigma_{R}}{\sqrt{r_{R}}} Z_{R}}{\sqrt{\frac{\sigma_{E}^{2}}{r_{E}}+\frac{\sigma_{R}^{2}}{r_{R}}}}, \frac{\frac{\sigma_{R}}{\sqrt{r_{R}}} Z_{R}-\frac{\sigma_{P}}{\sqrt{r_{P}}} Z_{P}}{\sqrt{\frac{\sigma_{R}^{2}}{r_{R}}+\frac{\sigma_{P}^{2}}{r_{P}}}}\right\}
$$

Finally, because the distribution of $T_{B}^{P I}$ and its limiting distribution are both continuous, so $q_{1-\alpha}^{B} \rightarrow q_{1-\alpha}$ as $n \rightarrow \infty$, where $q_{1-\alpha}$ be the $1-\alpha$ percentile point of $T$. This completes the proof.

\section{References}

1. Tango, T. Equivalence test and confidence interval for the difference in proportions for the paired-sample design. Stat. Med. 1998, 17, 891-908. [CrossRef]

2. Tang, N.S.; Tang, M.L.; Chan, S.F. On tests of equivalence via non-unity relative risk for matached-pair design. Stat. Med. 2003, 22, 1217-1233. [CrossRef]

3. Tang, M.L.; Tang, N.S.; Chan, S.F. Confidence interval construction for proportion difference in small-sample paired studies. Stat. Med. 2005, 24, 3565-3579. [CrossRef]

4. ICH E10. Harmonised Tripartite Guideline. Choice of Control Group and Related Issues in Clinical Trials, International Conference on Harmonisation of Technical Requirements for Registration of Pharmaceuticals for Human Use; ICH Steering Committee: Geneva, Switzerland, 2000.

5. Pigeot, I.; Schafer, J.; Rohmel, J.; Hauschke, D. Assessing non-inferiority of a new treatment in a three-arm clinical trial including a placebo. Stat. Med. 2003, 22, 883-899. [CrossRef]

6. Tang, M.L.; Tang, N.S. Tests of Noninferiority via Rate Difference for Three-Arm Clinical Trials with Placebo. J. Biopharm. Stat. 2004, 14, 337-347. [CrossRef]

7. Tang, N.S.; Yu, B.; Tang, M.L. Testing non-inferiroty of a new treatment in three-arm clinical trials with binary endpoints. BMC Med. Res. Methodol. 2014, 14, 134. [CrossRef] [PubMed]

8. Hida, E.; Tango, T. On the three-arm non-inferiority trial including a placebo with a prespecified margin. Stat. Med. 2011, 30, $224-231$. [CrossRef] [PubMed]

9. Kwong, K.S.; Cheung, S.H.; Hayter, A.J.; Wen, M. Extension of three-arm non-inferiority studies to trials with multiple new treatments. Stat. Med. 2012, 31, 2833-2843. [CrossRef] [PubMed]

10. Mütze, T.; Konietschke, F.; Munk, A.; Friede, T. A studentized permutation test for three-arm trials in the 'gold standard' design. Stat. Med. 2017, 36, 883-898. [CrossRef] [PubMed]

11. Newcombe, R.G. Interval estimation for the difference between independent proportions: Comparison of eleven methods. Stat. Med. 1998, 17, 873-890. [CrossRef]

12. Li, H.Q.; Tang, M.L.; Poon, W.Y.; Tang, N.S. Confidence Intervals for Difference Between Two Poisson Rates. Commun. Stat.-Simul. Comput. 2011, 40, 1478-1493. [CrossRef] 
13. Tang, N.S.; Qiu, S.F.; Tang, M.L.; Zou, G.Y.; Yu, D. Simultaneous Confidence Intervals of Risk Differences in Stratified Paired Designs. J. Biopharm. Stat. 2013, 23, 361-377. [CrossRef]

14. Tang, N.S.; Li, H.Q.; Tang, M.L.; Li, J. Confidence interval construction for the difference between two correlated proportions with missing observations. J. Biopharm. Stat. 2016, 26, 323-338. [CrossRef] [PubMed]

15. Zhang, Y.Q.; Tang, N.S. Confidence intervals of the difference between areas under two ROC curves in matched-pair experiments. Stat. Med. 2020, 39, 2621-2638. [CrossRef]

16. Weerahandi, S. Generalized confidence intervals. J. Am. Stat. Assoc. 1993, 88, 899-905. [CrossRef]

17. Hanning, J.; Iyer, H.; Patterson, P. Fiducial generalized condifence intervals. J. Am. Stat. Assoc. 2006, 101, 254-269. [CrossRef]

18. Hanning, J.; Iyer, H.; Lai, R.C.S.; Lee, T. Generalized fiducial inference: A review and new results. J. Am. Stat. Assoc. 2016, 111, 1346-1361. [CrossRef]

19. Schaarschmidt, F. Simultaneous confidence intervals for multiple comparisons among expected values of log-normal variables. Comput. Stat. Data Anal. 2013, 58, 265-275. [CrossRef]

20. Gamalo, M.A.; Muthukumarana, S.; Ghosh, P.; Tiwari, R.C. A generalized p-value approach for assessing noninferiority in a three-arm trial. Stat. Methods Med. Res. 2013, 22, 261-277. [CrossRef] [PubMed]

21. Abdel-Karim, A. Applications of Generalized Inference. Doctoral Dissertation, Colorado State University, Fort Collins, CO, USA, 2005.

22. Sadooghi-Alvandi, S.M.; Malekzadeh, A. Simultaneous confidence intervals for ratios of means of several lognormal distributions: A parametric bootstrap approach. Comput. Stat. Data Anal. 2014, 69, 133-140. [CrossRef]

23. Howe, W.G. Approximate confidence limits on the mean of $X+Y$ where $X$ and $Y$ are two tabled independent random variables. $J$. Am. Stat. Assoc. 1974, 69, 789-794. [CrossRef]

24. Ghosh, S.; Ghosh, S.; Tiwari, R.C. Bayesian approach for assessing non-inferiority in a three-arm trial with pre-specified margin. Stat. Med. 2016, 35, 695-708. [CrossRef] [PubMed] 